Utah State University

DigitalCommons@USU

Mechanical and Aerospace Engineering Student Mechanical and Aerospace Engineering Student Publications and Presentations

$1-4-2021$

\title{
Sensitivity and Estimation of Flying-Wing Aerodynamic, Propulsion, and Inertial Parameters Using Simulation
}

Jaden Thurgood

Utah State University, jadenthurgood7@gmail.com

Douglas F. Hunsaker

Utah State University, doug.hunsaker@usu.edu

Follow this and additional works at: https://digitalcommons.usu.edu/mae_stures

Part of the Aerospace Engineering Commons, and the Mechanical Engineering Commons

\section{Recommended Citation}

Thurgood, J. W., and Hunsaker, D. F., "Sensitivity and Estimation of Flying-Wing Aerodynamic, Propulsion, and Inertial Parameters Using Simulation," AIAA SciTech Forum Virtual Event, January 2021, AIAA-2021-1528, DOI: 10.2514/6.2021-1528

This Conference Paper is brought to you for free and open access by the Mechanical and Aerospace Engineering Student Research at DigitalCommons@USU. It has been accepted for inclusion in Mechanical and Aerospace Engineering Student Publications and Presentations by an authorized administrator of DigitalCommons@USU. For more information, please contact digitalcommons@usu.edu.

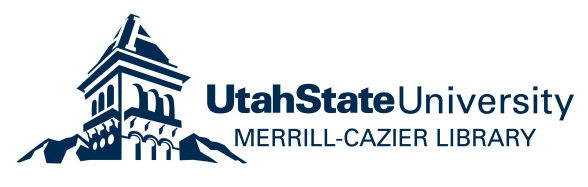




\title{
Sensitivity and Estimation of Flying-Wing Aerodynamic, Propulsion, and Inertial Parameters Using Simulation
}

\author{
Jaden W. Thurgood, ${ }^{*}$ and Douglas F. Hunsaker ${ }^{\dagger}$ \\ Utah State University, Logan, Utah, 84322, U.S.A.
}

\begin{abstract}
This paper explores the difficulties of aircraft system identification, specifically parameter estimation, for a rudderless aircraft. A white box method is used in conjunction with a nonlinear six degree-of-freedom aerodynamic model for the equations of motion in order to estimate 33 parameters that govern the aerodynamic, inertial, and propulsion forces within the mathematical model. The analysis is conducted in the time-domain of system identification. Additionally, all the parameters are estimated using a single flight rather than a series of shorter flights dedicated to estimating specific sets of parameters as is typically done. A final flight plan is developed with a mixture of lateral maneuvers interspersed throughout the flight to accentuate the significance of the lateral parameters during estimation. Certain parameters were ill-conditioned for parameter estimation using the mathematical model and final flight plan derived in this paper. The gradient-based optimization technique used in the estimation algorithm struggled to accurately estimate all 33 in a single flight due to the abundance of local minima within the solution space. The results of this work may provide a few insights for parameter estimation. First, to understand why system identification is performed the way it is currently done through multiple different flight maneuvers. Second, to gain some visual insight to the behavior of the nonlinear six degree-of-freedom aerodynamic model that describes the motion of fixed wing aircraft. This work may also be helpful in determining which parameters might likely be estimated together and which may struggle due to coupled dynamic relations within the mathematical model.
\end{abstract}

\section{Nomenclature}

a $\quad=$ density ratio constant

$b_{w} \quad=$ wingspan

$C_{D} \quad=$ drag coefficient in the wind coordinate system

$C_{D o} \quad=$ drag coefficient at the reference flight condition

$C_{D O}$

$=$ drag coefficient at zero angle of attack, rotational rate, and elevator deflection

$=$ linear coefficient in the parabolic relation for the drag coefficient as a function of the lift coefficient

$=$ quadratic coefficient in the parabolic relation for the drag coefficient as a function of the lift coefficient

$=$ quadratic coefficient in the relation for the drag coefficient as a function of the side-force coefficient

$=$ drag coefficient of the propulsion system

$=$ change in drag coefficient with respect to dimensionless pitch rate

$C_{D, \bar{q}}$

$C_{D, \delta_{e}}$

$C_{L}$

$C_{L o}$

$=$ change in drag coefficient with respect to elevator deflection

$=$ lift coefficient in the wind coordinate systems

$=$ lift coefficient at the reference flight condition

$C_{L_{0}} \quad=$ lift coefficient at zero angle of attack, rotational rate, and elevator deflection

$C_{L, \alpha} \quad=$ lift slope - change in lift coefficient with respect to angle of attack

$C_{L, \bar{q}} \quad=$ change in lift coefficient with respect to dimensionless pitch rate

$C_{L, \delta_{e}} \quad=$ change in lift coefficient with respect to elevator deflection

$C_{\ell} \quad=$ rolling moment coefficient in the body-fixed coordinate system

$C_{\ell, \beta} \quad=$ change in rolling moment coefficient with respect to sideslip angle

$C_{\ell, \bar{p}} \quad=$ change in rolling moment coefficient with respect to dimensionless roll rate

$C_{\ell, \bar{r}} \quad=$ change in rolling moment coefficient with respect to dimensionless yaw rate

$C_{\ell, \delta_{a}} \quad=$ change in rolling moment coefficient with respect to aileron deflection

${ }^{*}$ Graduate Research Assistant, Mechanical and Aerospace Engineering Dept., 4130 Old Main Hill, AIAA Member

$\dagger$ Assistant Professor, Mechanical and Aerospace Engineering Dept., 4130 Old Main Hill, AIAA Senior Member 


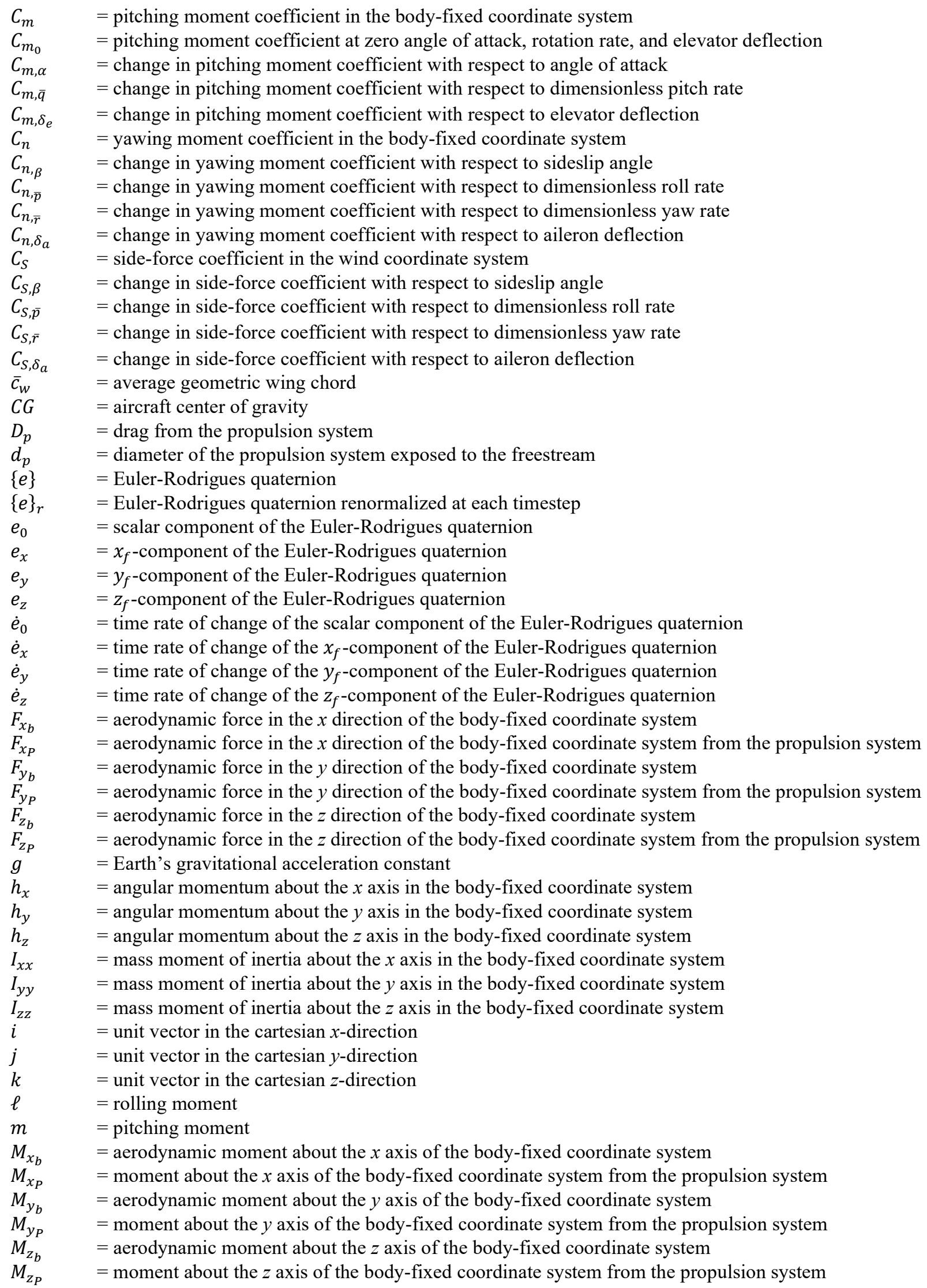









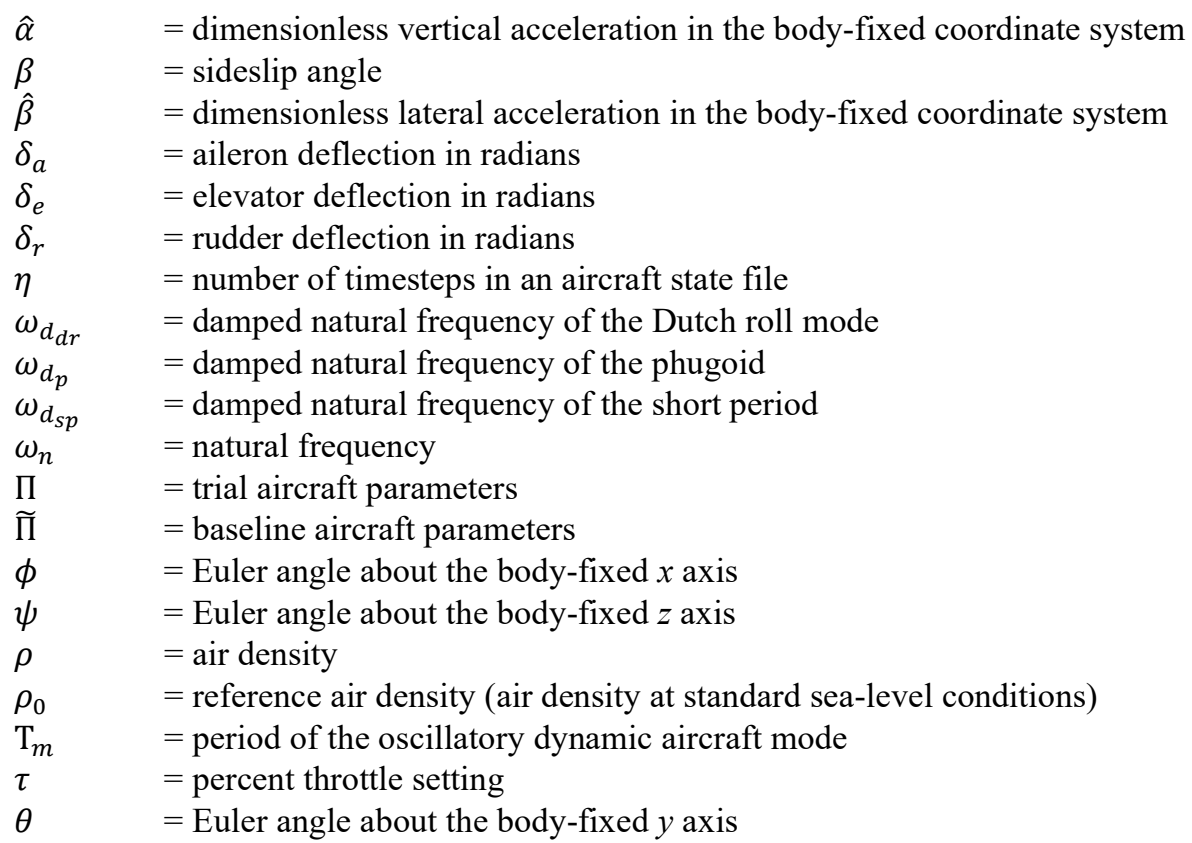

\section{Introduction}

$\mathrm{T}$ he demand for small unmanned aircraft systems (SUAS) that can autonomously carry out civilian and commercial purposes, such as photography and mapping, is growing. This desired autonomy demands improved development of guidance, navigation, and control (GNC) algorithms which rely on a robust understanding and implementation of the mathematical model that approximates the dynamics of the aircraft [1]. One way of developing and refining these algorithms and dynamic models is through system identification. System identification and simulation play a large role in the rapid design and testing of SUAS. System identification allows for the rapid development of a mathematical model that approximates the dynamics of a system and its associated parameters. This mathematical model can vary in complexity and accuracy depending on the needs of the project. Flight simulation provides a low-cost approach to repetitive testing without the risk of material and time losses in the event of a crash.

System identification may be defined as the iterative process of determining, or refining, mathematical models of a dynamic system by analyzing the relationship between inputs and the measured outputs of the dynamic system [2, 3]. One way to think of system identification is that it is the inverse of simulation. For example, in simulation, we start with first principles to derive a set of equations that will govern the behavior of a given system. Then we run test cases through the simulation to get predicted outcomes based on the initial conditions and any inputs that may affect the system. We can then compare the predicted outcomes from the simulation with test data to determine the validity of our model. However, system identification works backwards relative to the simulation process. In the world of system identification, we start with recorded test data and inputs to a given model and seek to either develop a mathematical model or estimate parameters within a pre-determined model. The two processes work hand in hand to iteratively improve the other. Results from simulation improve system identification and vice versa. The process in Fig. 1 gives an outline of the flow of the simulation and system identification processes.

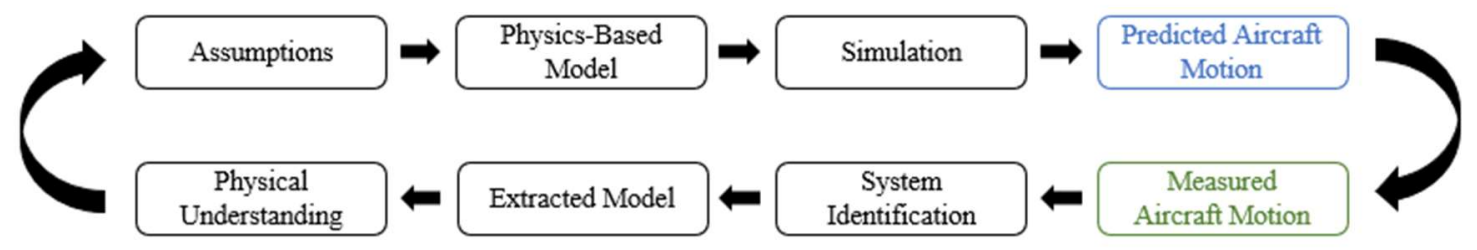

Fig. 1 Relationship between simulation and system identification.

In the world of aircraft system identification there are two main challenges to accurately predicting the dynamic behavior of an aircraft. These challenges are the identification of a mathematical model, and the estimation of the parameters within that mathematical model [3]. These two challenges are commonly described as "black box" and 
"gray box" models. Black box models seek to solve the challenge of identifying a reliable mathematical model and parameter estimation, while gray box models use a pre-constructed model that can be based on physical insight or experimental data [4]. Gray box models typically solve the challenge of parameter estimation, but they are also used for refining an existing mathematical model, as described by Venkataraman [1]. However, some authors make a further distinction by defining models that are only tasked with parameter estimation as white box models [5].

This work employs a white box model by starting with first principles to build a nonlinear, six degrees-of-freedom (DOF) model for the rudderless, fixed-wing SUAS described in Section IV. Therefore, the challenge in this work is the estimation of the parameters within the model. Parameter estimation can be conducted in several methods such as: equation error methods, output error methods, and filter error methods [5]. In this paper, we implement an output error system through an iterative least-squares method in conjunction with gradient-based optimization techniques.

There are several texts dedicated to the process of modeling the mechanics of rigid-body aircraft $[6,7]$. Similarly, there are several resources for applying system identification techniques to fixed-wing aircraft $[2,5,8]$. However, literature for system identification of rudderless SUAS is limited due to the difficulty of lateral parameter identification. During system identification, certain flight maneuvers are performed to accentuate certain dynamic modes of the aircraft in the flight test data. The lack of a rudder on the aircraft makes it difficult to excite certain lateral modes of the aircraft. The work that has been done for these types of aircraft include the work on the ICE/SACCON aircraft $[8,9]$ and the X-56A flexible aircraft $[10,11]$. The work on each of these aircraft is being conducted in the frequency domain. However, few have approached this problem in the time-domain. The parameter sensitivity and estimation in this work is conducted in the time-domain.

Until recently, system identification in the time and frequency domains were seen as competing methods. The frequency domain dominated the literature up through the 1960's. Then, from the 1960's to the 1980's more interest in the time-domain led to techniques and literature for that method. Since then, the frequency domain has regained interest and today both methods are used. Both, however, have advantages and disadvantages that generally complement each other [12]. The frequency domain refers to the space where mathematical functions are conveyed in terms of frequency, instead of time. The time-domain tends to be a little more intuitive, since humans tend to think in terms of time processes. For example, where a time-domain graph might display some arbitrary change over a given time period, the frequency domain graph would display an arbitrary change over a given range of frequencies.

The work in this research was conducted in the time-domain which lends to a couple of advantages. If a model is known a priori and further refinement about that model is desired, the time-domain easily allows the user to choose the model to be used. In the frequency domain, the model, unless derived in the frequency domain, would need to be transformed, which can be laborious and difficult. Moreover, the parameters of a given mathematical model have a physical meaning in the time-domain. In the frequency domain, these appear more abstract and need to be transformed back to the time-domain to take on a physical meaning. Finally, model validation is typically only done in the timedomain. Therefore, having a model in the time-domain allows for a more rapid transition to model validation [12].

As early as 1989, Tischler et al. [13] showed that both the time and frequency domain system identification approaches were able to determine adequate six DOF rigid-body models. They also showed that the two methods were similar in their parameter estimation of the stability, damping, and control derivatives. Unless the process requires the ability to model data with very wide frequency ranges, continuous-time models, or if a subsystem is being modeled, then the choice between the time and frequency domain may be made based on familiarity or availability of resources $[14,15]$.

This paper presents a method of understanding the sensitivity and estimation of aircraft flight parameters by employing a system identification technique using flight simulation as the test bed in lieu of a physical prototype aircraft conventionally used for collecting experimental data. This lends to a cheaper initial pass at collecting data for aircraft system identification and allows the user to tailor the output of the simulator to the outputs needed for system identification. This contrasts with a traditional setup where sensor data from the prototype aircraft must be manipulated through post-processing techniques.

The white box models in system identification are responsible for estimating the parameters within the mathematical model. Typically, parameter estimation is done through a series of independent flight maneuvers that are specifically designed to identify two to three parameters at a time. The recorded flight data is relatively short, and the maneuver is repeated multiple times to collect sets of data for stochastic considerations during estimation. This process is tedious and lengthy. In order to estimate the 33 parameters in this paper, a conventional approach may need as many as 15-20 unique flight maneuvers that are repeated multiple times, resulting in 45-60 sets of flight data to keep track of. The work presented in this paper is unique in that it attempts to combine the necessary maneuvers into 
one larger set of flight data. The final flight test data is run through the estimation algorithm where all 33 parameters are estimated in a single run.

Throughout this document, we will refer to two separate aircraft. The baseline aircraft and the trial aircraft. The baseline aircraft will be considered the aircraft with the true aerodynamic parameters, while the trial aircraft will be the aircraft that is tested, compared, and adjusted relative to the baseline aircraft. The baseline aircraft uses Phillips and Snyder's adapted lifting-line method [16] in conjunction with Reid and Hunsaker's sweep corrections [17] to define the aerodynamic characteristics (stability, damping, and control derivatives) of the aircraft. The purpose of the sensitivity analysis is to determine the longitudinal and lateral flight maneuvers necessary for accurately estimating the aircraft parameters. The approach consists of running a simulated flight of the baseline aircraft and recording the states of that flight.

Next, the baseline aircraft model parameters are estimated by running trial aircraft flights through a steepest descent optimization scheme. The optimization technique adjusts the parameters of the trial aircraft model until the states of the trial flights converge to the states of the baseline flight. Although, this approach of using a flight simulator allows for frequency and time-domain approaches, we have employed a time-domain approach coupled with a white box model to estimate the parameters of the nonlinear 6-DOF model.

\section{Flight Simulation}

This section covers how the aircraft aerodynamics were derived and simulated. The first part presents a brief review of aerodynamic forces and moments. The second part defines the coordinate systems and the transformations of an arbitrary vector from one coordinate system to another. The third part details the derivation of the aerodynamic model from the forces and moments acting on the aircraft which are used in the equations of motion that govern the dynamics of the aircraft. The fourth part describes how the propulsive forces and moments on the aircraft are modeled. Finally, the last part couples the aerodynamic and propulsion models with Newtons equations of motion.

Within the literature of system identification and flight testing, the stall region is generally avoided. In order to avoid modeling stall, we only operate in configurations that result in nearly linear behavior. Developing an accurate stall model for an aircraft is not a trivial task, nor is it the topic of this work. If parameters in the stall model are needed, a separate model specific to the stall regime can be used and then the parameter results from the linear and stall regions can be combined later [5].

\section{A. Aerodynamic Forces and Moments Review}

A brief definition of the forces and moments acting on an aircraft are presented here. The aerodynamic forces acting on an aircraft are referred to as lift, drag, and side-force. Drag on an aircraft acts in the direction of the freestream velocity, or opposite the direction the aircraft is traveling. The lift on an aircraft acts perpendicular to the freestream velocity vector, and the side-force acts in the direction of the lift vector crossed into the drag vector shown in Fig. 2. This section omits the forces and moments from propulsion as that will be addressed more specifically in Section F. The aerodynamic moments acting on an aircraft in the body-fixed coordinate system are referred to as roll, pitch, and yaw and are denoted $\ell, m$, and $n$ respectively.

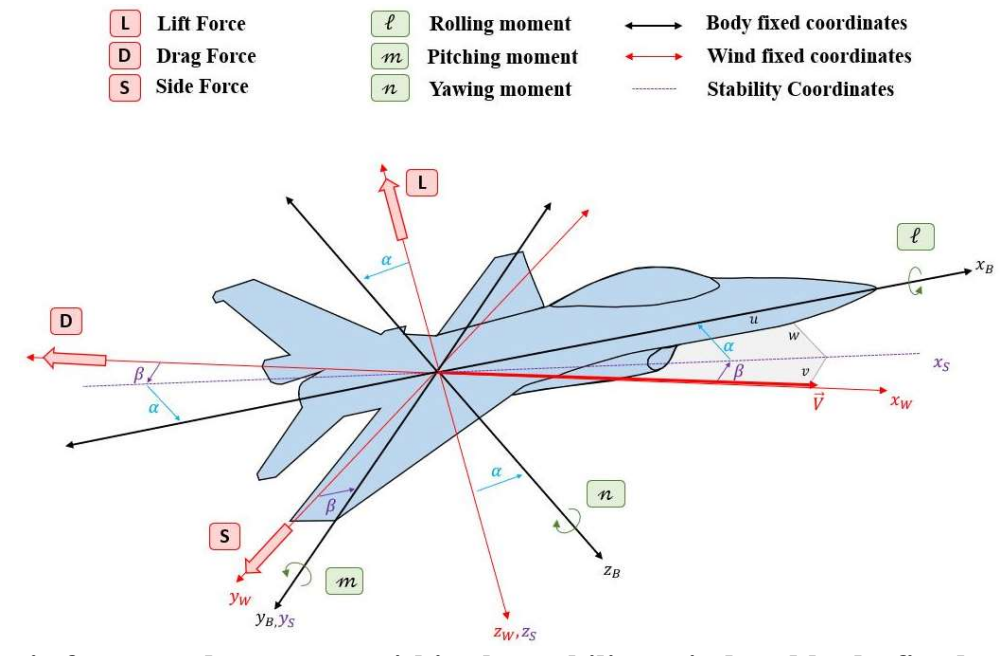

Fig. 2 Aerodynamic forces and moments within the stability, wind and body-fixed coordinate systems. 


\section{B. Coordinate System Transformations}

The coordinate systems used throughout the following aerodynamic model derivation are the stability, body-fixed, and wind coordinate systems. The transformations in Fig. 3 shows a simple representation of the three coordinate systems and how they are related to one another through aerodynamic angle rotations $\alpha$ and $\beta$. The aerodynamic angles, $\alpha$ and $\beta$ are defined as

$$
\begin{aligned}
& \alpha \equiv \tan ^{-1}\left(\frac{w}{u}\right) \\
& \beta \equiv \sin ^{-1}\left(\frac{v}{V}\right)
\end{aligned}
$$

where $u, v$, and $w$ are the body-fixed velocities of the aircraft and $V$ is the total velocity of the aircraft. Here we use the traditional definition for the sideslip angle $\beta$ as opposed to the flank angle, $\beta_{f}$, presented by Klein and Morelli [18]. It is important to be aware how each author defines the sideslip angle and more important to be consistent with the application. For simplicity, $\sin \alpha$ will be denoted as $s_{\alpha}$ and $\cos \alpha$ will be denoted as $c_{\alpha}$ throughout the remainder of the paper. The terms $\sin \beta$ and $\cos \beta$ will be denoted as $s_{\beta}$ and $c_{\beta}$.

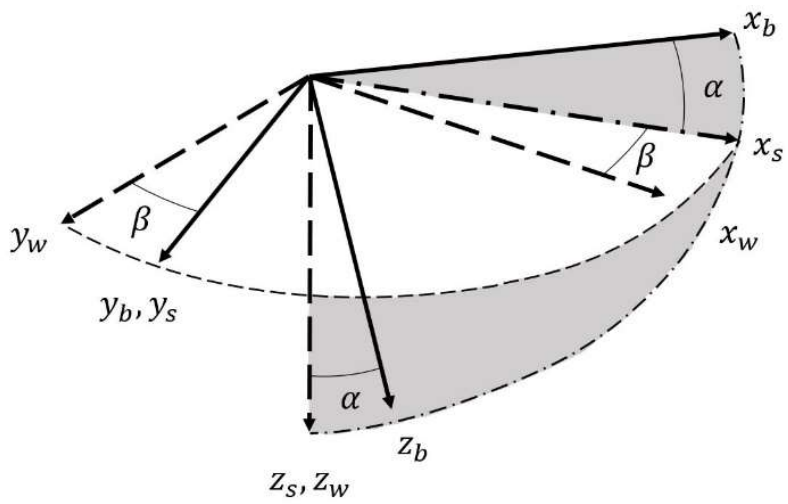

Fig. 3 Rotation from the wind axes to the stability and body-fixed axes through $\alpha$ and $\beta$.

Often in simulation, testing, and data collection, some information may be available in one coordinate system with other information in another coordinate system. This mismatch of coordinate systems necessitates the transformation of information in one coordinate system to its equivalent value in another coordinate system. This is particularly important because whereas aerodynamic forces are generally evaluated in either the stability or wind coordinates in most of the literature [7], equations of motion that govern an aircraft's dynamic behavior are typically written in the body-fixed coordinate system. Therefore, we generally use coordinate transformations to express the aerodynamic forces acting in the stability or wind coordinate system as equivalent forces in the body-fixed coordinate system.

The stability coordinate system is found by a rotation about the body-fixed $y$-axis through some angle of attack $\alpha$. It can be seen from Fig. 3 that the transformation of an arbitrary vector from the body-fixed to stability coordinate system is

$$
\left\{\begin{array}{l}
v_{x_{s}} \\
v_{y_{s}} \\
v_{z_{s}}
\end{array}\right\}=\left[\begin{array}{ccc}
c_{\alpha} & 0 & s_{\alpha} \\
0 & 1 & 0 \\
-s_{\alpha} & 0 & c_{\alpha}
\end{array}\right]\left\{\begin{array}{l}
v_{x_{b}} \\
v_{y_{b}} \\
v_{z_{b}}
\end{array}\right\}
$$

and the inverse transformation is

$$
\left\{\begin{array}{l}
v_{x_{b}} \\
v_{y_{b}} \\
v_{z_{b}}
\end{array}\right\}=\left[\begin{array}{ccc}
c_{\alpha} & 0 & -s_{\alpha} \\
0 & 1 & 0 \\
s_{\alpha} & 0 & c_{\alpha}
\end{array}\right]\left\{\begin{array}{l}
v_{x_{s}} \\
v_{y_{s}} \\
v_{z_{s}}
\end{array}\right\}
$$

Similarly, the wind coordinate system is found by a rotation about the stability $z$-axis through some sideslip angle $\beta$. 
The transformation from a vector in the stability coordinate system to the wind coordinate system is

$$
\left\{\begin{array}{l}
v_{x_{w}} \\
v_{y_{w}} \\
v_{z_{w}}
\end{array}\right\}=\left[\begin{array}{ccc}
c_{\beta} & s_{\beta} & 0 \\
-s_{\beta} & c_{\beta} & 0 \\
0 & 0 & 1
\end{array}\right]\left\{\begin{array}{l}
v_{x_{s}} \\
v_{y_{s}} \\
v_{z_{s}}
\end{array}\right\}
$$

and the inverse transformation is

$$
\left\{\begin{array}{l}
v_{x_{s}} \\
v_{y_{s}} \\
v_{z_{s}}
\end{array}\right\}=\left[\begin{array}{ccc}
c_{\beta} & -s_{\beta} & 0 \\
s_{\beta} & c_{\beta} & 0 \\
0 & 0 & 1
\end{array}\right]\left\{\begin{array}{l}
v_{x_{w}} \\
v_{y_{w}} \\
v_{z_{w}}
\end{array}\right\}
$$

Using a combination of Eqs. (3) through (6), it can also be shown that the transformation from a vector in the wind coordinate system to the body-fixed system is

$$
\left\{\begin{array}{l}
v_{x_{b}} \\
v_{y_{b}} \\
v_{z_{b}}
\end{array}\right\}=\left[\begin{array}{ccc}
c_{\alpha} c_{\beta} & -c_{\alpha} s_{\beta} & -s_{\alpha} \\
s_{\beta} & c_{\beta} & 0 \\
s_{\alpha} c_{\beta} & -s_{\alpha} s_{\beta} & c_{\alpha}
\end{array}\right]\left\{\begin{array}{l}
v_{x_{w}} \\
v_{y_{w}} \\
v_{z_{w}}
\end{array}\right\}
$$

and the inverse relationship is

$$
\left\{\begin{array}{l}
v_{x_{w}} \\
v_{y_{w}} \\
v_{z_{w}}
\end{array}\right\}=\left[\begin{array}{ccc}
c_{\alpha} c_{\beta} & s_{\beta} & s_{\alpha} c_{\beta} \\
-c_{\alpha} s_{\beta} & c_{\beta} & -s_{\alpha} s_{\beta} \\
-s_{\alpha} & 0 & c_{\alpha}
\end{array}\right]\left\{\begin{array}{l}
v_{x_{b}} \\
v_{y_{b}} \\
v_{z_{b}}
\end{array}\right\}
$$

As an important note, there is another coordinate system that is commonly used in aircraft simulation known as the Earth-fixed coordinate system. This coordinate system is defined by the $x_{f}-y_{f}$ plane being normal to the local gravitational vector. The $x_{f}$-axis points north and the $y_{f}$-axis points east. The $z_{f}$-axis points down towards the center of the Earth, parallel to the Earths gravitational vector. The transformation of information from the body-fixed to Earth-fixed coordinate systems is presented in Sec G. 2.

\section{Non-Dimensional Aerodynamic Forces}

In deriving the complete set of equations used to model the aerodynamic forces, we assume angles of attack below stall, small sideslip angles, and small control-surface deflections. With this assumption, small-disturbance approximations may be used to formulate linear equations for the non-dimensional aerodynamic forces of lift, sideforce, and drag. Assuming the drag force is a nearly parabolic function of lift and side-force, the small-disturbance approximations for the force coefficients on an aircraft in the wind coordinate system are

$$
\begin{gathered}
C_{L}=C_{L_{0}}+C_{L, \alpha} \alpha+C_{L, \beta} \beta+C_{L, \bar{p}} \bar{p}+C_{L, \bar{q}} \bar{q}+C_{L, \bar{r}} \bar{r}+C_{L, \widehat{\alpha}} \hat{\alpha}+C_{L, \widehat{\beta}} \hat{\beta}+C_{L, \delta_{e}} \delta_{e} \\
C_{S}=C_{S, \alpha} \alpha+C_{S, \beta} \beta+C_{S, \bar{p}} \bar{p}+C_{S, \bar{q}} \bar{q}+C_{S, \bar{r}} \bar{r}+C_{S, \widehat{\alpha}} \hat{\alpha}+C_{S, \widehat{\beta}} \hat{\beta}+C_{S, \delta_{a}} \delta_{a} \\
C_{D}=C_{D 0}+C_{D 1} C_{L}+C_{D 2} C_{L}^{2}+C_{D 3} C_{S}^{2}+C_{D, \bar{p}} \bar{p}+C_{D, \bar{q}} \bar{q}+C_{D, \bar{r}} \bar{r}+C_{D, \widehat{\alpha}} \hat{\alpha}+C_{D, \widehat{\beta}} \hat{\beta}+C_{D, \delta_{e}} \delta_{e}
\end{gathered}
$$

Here, $C_{L_{0}}$ is the lift coefficient at condition of zero angle of attack, zero sideslip, zero rotational rates, zero translational acceleration, and zero control surface deflections. The drag terms $C_{D 0}, C_{D 1}, C_{D 2}$, and $C_{D 3}$ in Eq. (11) are coefficients used to model the quadratic dependence of the drag on the lift and side-force. There are a handful of methods for determining these four drag coefficient terms. One method depends on knowing the lift and drag and the first and second derivatives of drag at a given reference condition and then analytically estimating the coefficients. However, perhaps a more straightforward and accurate method is to measure the lift and drag at several angles of attack and sideslip angles and then implement a least-squares approach to estimating the coefficients for a desired aircraft configuration. This method is used to determine the drag terms in Table 2 analytically by calculating the lift and drag on the aircraft at incremental angles of attack around zero using a tool called MachUpX. MachUpX is a python-based aerodynamic tool which uses Phillips and Snyder's adapted lifting-line method [16] as well as Reid and Hunsaker's sweep corrections [17]. The coefficients are then estimated through a least-squares best-fit approach. The last drag term, $C_{D 3}$, is approximated in a similar manner using side-force and drag forces on the aircraft at varying sideslip

\footnotetext{
* https://github.com/usuaero/MachUpX
} 
angles around zero. The remaining terms in Eq. (9) through (11) are the nondimensional, roll rate, pitch rate, and yaw rate, defined as

$$
\begin{gathered}
\bar{p} \equiv \frac{p b_{w}}{2 V} \\
\bar{q} \equiv \frac{q \bar{c}_{w}}{2 V} \\
\bar{r} \equiv \frac{r b_{w}}{2 V}
\end{gathered}
$$

the nondimensional vertical and lateral translational accelerations are defined, respectively, as

$$
\begin{gathered}
\hat{\alpha} \equiv \frac{\dot{\alpha} \bar{c}_{w}}{2 V} \\
\hat{\beta} \equiv \frac{\dot{\beta} b_{w}}{2 V}
\end{gathered}
$$

and the elevator, aileron, and rudder control surface deflections, in radians, are respectively given by $\delta_{e}, \delta_{a}$, and $\delta_{r}$. The aircraft average geometric wing chord and wingspan are defined as $\bar{c}_{w}$ and $b_{w}$ respectively.

\section{Non-Dimensional Aerodynamic Moments}

The set of equations used to model the aerodynamic moments on an aircraft also assume angles of attack below stall, small sideslip angles, and small control-surface deflections. The linear small-disturbance approximations for the non-dimensional aerodynamic moments of roll, pitch, and yaw on the aircraft in the body-fixed coordinate system are

$$
\begin{gathered}
C_{\ell}=C_{\ell, \alpha} \alpha+C_{\ell, \beta} \beta+C_{\ell, \bar{p}} \bar{p}+C_{\ell, \bar{q}} \bar{q}+C_{\ell, \bar{r}} \bar{r}+C_{\ell, \widehat{\alpha}} \hat{\alpha}+C_{\ell, \widehat{\beta}} \hat{\beta}+C_{\ell, \delta_{a}} \delta_{a} \\
C_{m}=C_{m_{0}}+C_{m, \alpha} \alpha+C_{m, \beta} \beta+C_{m, \bar{p}} \bar{p}+C_{m, \bar{q}} \bar{q}+C_{m, \bar{r}} \bar{r}+C_{m, \widehat{\alpha}} \hat{\alpha}+C_{m, \widehat{\beta}} \hat{\beta}+C_{m, \delta_{e}} \delta_{e} \\
C_{n}=C_{n, \alpha} \alpha+C_{n, \beta} \beta+C_{n, \bar{p}} \bar{p}+C_{n, \bar{q}} \bar{q}+C_{n, \bar{r}} \bar{r}+C_{n, \widehat{\alpha}} \hat{\alpha}+C_{n, \widehat{\beta}} \hat{\beta}+C_{n, \delta_{a}} \delta_{a}
\end{gathered}
$$

Thankfully, the linear aerodynamic equations in Eq. (9) through (11) and Eq. (17) through (19) are drastically simplified for most common fixed-wing aircraft. For example, most aircraft are nearly symmetrical. This means that at small sideslip angles, many of the lateral aerodynamic derivatives with respect to the longitudinal angle of attack and elevator deflections are nearly zero, i.e.,

$$
\begin{aligned}
& C_{S, \alpha} \cong C_{S, \bar{q}} \cong C_{S, \widehat{\alpha}} \cong C_{S, \delta_{e}} \cong 0 \\
& C_{\ell, \alpha} \cong C_{\ell, \bar{q}} \cong C_{\ell, \widehat{\alpha}} \cong C_{\ell, \delta_{e}} \cong 0 \\
& C_{n, \alpha} \cong C_{n, \bar{q}} \cong C_{n, \widehat{\alpha}} \cong C_{n, \delta_{e}} \cong 0
\end{aligned}
$$

Similarly, symmetry allows us to simplify some of the longitudinal aerodynamic derivatives with respect to the sideslip angle and lateral control surfaces, i.e.,

$$
\begin{gathered}
C_{L, \beta} \cong C_{L, \bar{p}} \cong C_{L, \bar{r}} \cong C_{L, \widehat{\beta}} \cong C_{L, \delta_{a}} \cong C_{L, \delta_{r}} \cong 0 \\
C_{D, \bar{p}} \cong C_{D, \bar{r}} \cong C_{D, \widehat{\beta}} \cong C_{D, \delta_{a}} \cong C_{D, \delta_{r}} \cong 0 \\
C_{m, \beta} \cong C_{m, \bar{p}} \cong C_{m, \bar{r}} \cong C_{m, \widehat{\beta}} \cong C_{m, \delta_{a}} \cong C_{m, \delta_{r}} \cong 0
\end{gathered}
$$

The approximations in Eq. (21) come from the fact that changes in the lateral terms have an identical effect on the longitudinal forces and moments, whether they are positive or negative. For example, the change in drag produced from a positive aileron deflection is approximately the same as the change in drag produced from a negative aileron deflection because we linearly represent the model relative to the configuration of no control surface deflection. 
Finally, determining the derivatives with respect to the translational accelerations in Eqs. (9) - (11) and Eqs. (17) - (19) is not trivial. Typically, these are obtained through an unsteady flow analysis, either in a wind tunnel or CFD model. Phillips [7] provides an analytical estimate for the change in lift and pitching moment with respect to the vertical acceleration. He further mentions that all the other changes in forces and moments with respect to the translational accelerations are zero or near zero. In this work, we assume that the change in lift and pitching moment with respect to the vertical acceleration are nearly zero. We also assume that the changes in side-force, rolling and yawing moments with respect to the lateral acceleration are nearly zero. This results in

$$
C_{L, \widehat{\alpha}} \cong C_{D, \widehat{\alpha}} \cong C_{S, \widehat{\beta}} \cong C_{\ell, \widehat{\beta}} \cong C_{m, \widehat{\alpha}} \cong C_{n, \widehat{\beta}} \cong 0
$$

\section{E. Aerodynamic Model for the Forces and Moments}

Applying the assumptions from Eqs. (20) - (22) to Eqs. (9) - (11) and Eqs. (17) - (19) results in the simplified equations

$$
\begin{gathered}
C_{L}=C_{L_{0}}+C_{L, \alpha} \alpha+C_{L, \bar{q}} \bar{q}+C_{L, \delta_{e}} \delta_{e} \\
C_{S}=C_{S, \beta} \beta+C_{S, \bar{p}} \bar{p}+C_{S, \bar{r}} \bar{r}+C_{S, \delta_{a}} \delta_{a} \\
C_{D}=C_{D 0}+C_{D 1} C_{L}+C_{D 2} C_{L}^{2}+C_{D 3} C_{S}^{2}+C_{D, \bar{q}} \bar{q}+C_{D, \delta_{e}} \delta_{e} \\
C_{\ell}=C_{\ell, \beta} \beta+C_{\ell, \bar{p}} \bar{p}+C_{\ell, \bar{r}} \bar{r}+C_{\ell, \delta_{a}} \delta_{a} \\
C_{m}=C_{m_{0}}+C_{m, \alpha} \alpha+C_{m, \bar{q}} \bar{q}+C_{m, \delta_{e}} \delta_{e} \\
C_{n}=C_{n, \beta} \beta+C_{n, \bar{p}} \bar{p}+C_{n, \bar{r}} \bar{r}+C_{n, \delta_{a}} \delta_{a}
\end{gathered}
$$

Because the aircraft being analyzed is a rudderless flying wing, any terms involving $\delta_{r}$ have been removed as well.

Now we have a model for the non-dimensional forces and moments acting on the aircraft. With the forces in the wind coordinate system, and the moments in the body-fixed coordinate system, we can formulate a system of equations that describes the dimensional forces and moments in the body-fixed coordinate system. The forces are given by

$$
\left\{\begin{array}{l}
F_{x_{b}} \\
F_{y_{b}} \\
F_{z_{b}}
\end{array}\right\}=\left\{\begin{array}{l}
F_{x_{P}} \\
F_{y_{P}} \\
F_{z_{P}}
\end{array}\right\}+\frac{1}{2} \rho V^{2} S_{w}\left\{\begin{array}{c}
C_{L} s_{\alpha}-C_{S} c_{\alpha} s_{\beta}-C_{D} c_{\alpha} c_{\beta} \\
C_{S} c_{\beta}-C_{D} s_{\beta} \\
-C_{L} c_{\alpha}-C_{S} s_{\alpha} s_{\beta}-C_{D} s_{\alpha} c_{\beta}
\end{array}\right\}
$$

where $F_{x_{P}}, F_{y_{P}}$, and $F_{z_{P}}$ are the body-fixed propulsion forces which are explained in the propulsion model, and $C_{L}, C_{S}$, and $C_{D}$ are normalized by the dynamic pressure and the wing area. The transformation equations from wind coordinates to body-fixed coordinate system given in Eq. (7) have been applied to the non-dimensional forces given in Eqs. (23) - (25).

Since the non-dimensional moment coefficient equations from Eqs. (26) - (28) are written in the body-fixed coordinate system, no transformation is needed. The dimensional moments acting on the aircraft are given by

$$
\left\{\begin{array}{l}
M_{x_{b}} \\
M_{y_{b}} \\
M_{z_{b}}
\end{array}\right\}=\left\{\begin{array}{l}
M_{x_{P}} \\
M_{y_{P}} \\
M_{z_{P}}
\end{array}\right\}+\frac{1}{2} \rho V^{2} S_{w}\left\{\begin{array}{l}
b_{w} C_{\ell} \\
\bar{c}_{w} C_{m} \\
b_{w} C_{n}
\end{array}\right\}
$$

where $M_{x_{P}}, M_{y_{P}}$, and $M_{z_{P}}$ are the body-fixed propulsion moment components explained in the following section and $C_{\ell}, C_{m}, C_{n}$, are normalized by the dynamic pressure and the wing area. 


\section{F. Propulsion Forces and Moments}

The propulsive forces and moments in Eq. (29) and (30) are outlined in the following set of equations. The forces of the propulsion system in the body-fixed coordinate system are given by

$$
\left\{\begin{array}{l}
F_{x_{P}} \\
F_{y_{P}} \\
F_{z_{P}}
\end{array}\right\}=T \boldsymbol{u}_{P}+D_{P} \boldsymbol{u}_{\infty}
$$

where $T$ and $D_{p}$ are the thrust and drag of the propulsion system respectively, and $\boldsymbol{u}_{P}$ and $\boldsymbol{u}_{\infty}$ are the unit vectors in the direction of thrust and the freestream respectively. The thrust of a propulsion system is often modeled using a quadratic function of velocity, which is also proportional to the throttle setting and air density. This can be written as

$$
T=\tau\left(\rho / \rho_{0}\right)^{a}\left(T_{0}+T_{1} V+T_{2} V^{2}\right)
$$

where $a$ is a constant related to the density ratio, and $T_{0}, T_{1}$, and $T_{2}$ are experimentally determined coefficients of the parabolic function that describes the thrust of the propulsion system on the aircraft [7]. These coefficients can be approximated by fitting Eq. (32) to predicted or measured thrust data for a given propulsion system.

The drag from the propulsion system is modeled as

$$
D_{P}=\frac{1}{2} \rho V^{2} S_{P} C_{D_{P}}
$$

where $S_{P}$ is the characteristic area of the propulsion system, and $C_{D_{P}}$ is the propulsion drag coefficient. The characteristic area of a propeller is given by

$$
S_{P}=\frac{\pi d_{P}^{2}}{4}
$$

where $d_{P}$ is the diameter of the propulsion system exposed to the freestream.

Typically, the propulsion drag coefficient can be approximated using predicted or measured drag data for the propulsion system. Finally, the moments of the propulsion system can be determined using

$$
\left\{\begin{array}{l}
M_{x_{P}} \\
M_{y_{P}} \\
M_{z_{P}}
\end{array}\right\}=\left\{\begin{array}{l}
r_{x_{P}} \\
r_{y_{P}} \\
r_{z_{P}}
\end{array}\right\} \times\left\{\begin{array}{l}
F_{x_{P}} \\
F_{y_{P}} \\
F_{z_{P}}
\end{array}\right\}
$$

where $r_{x_{P}}, r_{y_{P}}$, and $r_{z_{P}}$ make up the components of the vector from the aircraft CG to the propulsion system.

\section{G. Equations of Motion}

The ultimate intent of a flight simulator is to describe the state of the aircraft in time and space. Often referred to as the state vector, this array of data gives information such as translational and rotational velocities, as well as position and orientation in physical space. This section presents a system of equations that govern the dynamics of the aircraft. This system of equations follows Phillip's formulation [7]. Phillips uses quaternions for describing the orientation of an aircraft in space as opposed to traditional Euler angle, or direction-cosine matrices described in chapter 11 of his book [7]. The quaternion formulation offers lower computational cost and avoids gimbal lock. A brief overview of the method is given in the following subsection. The last part of this section outlines the equations of motion that describe the state of the aircraft and the timestep selection during simulation.

\section{Quaternion Overview}

The definition of the quaternion used in this paper is defined as

$$
\{\boldsymbol{e}\} \equiv e_{0}+e_{x} \boldsymbol{i}+e_{y} \boldsymbol{j}+e_{z} \boldsymbol{k}
$$

where $e_{0}, e_{x}, e_{y}$, and $e_{z}$ are scalar values and $\boldsymbol{i}, \boldsymbol{j}$, and $\boldsymbol{k}$ are the unit vectors in the cartesian $x, y$ and $z$ directions, respectively. 
The transformation from Euler angles $\phi, \theta$, and $\psi$ to the equivalent quaternion is

$$
\left\{\begin{array}{l}
e_{0} \\
e_{x} \\
e_{y} \\
e_{z}
\end{array}\right\}=\left\{\begin{array}{l}
c_{\phi / 2} c_{\theta / 2} c_{\psi / 2}+s_{\phi / 2} s_{\theta / 2} s_{\psi / 2} \\
s_{\phi / 2} c_{\theta / 2} c_{\psi / 2}-c_{\phi / 2} s_{\theta / 2} s_{\psi / 2} \\
c_{\phi / 2} s_{\theta / 2} c_{\psi / 2}+s_{\phi / 2} c_{\theta / 2} s_{\psi / 2} \\
c_{\phi / 2} c_{\theta / 2} c_{\psi / 2}-s_{\phi / 2} s_{\theta / 2} s_{\psi / 2}
\end{array}\right\}
$$

where $c_{\phi / 2}$ and $s_{\phi / 2}$ are the cosine and sine of half the bank angle $\phi$ respectively. The inverse of Eq. (37) is straightforward and provides a transformation from the quaternion to the Euler angles. Unfortunately, that transformation is subject to gimbal lock at $\theta= \pm \pi / 2$. In order to avoid issues with gimbal lock in the simulation, Phillips suggests an algorithm [7] written as

$$
\begin{aligned}
& \text { if }\left(e_{0} e_{y}-e_{x} e_{z}=0.5\right) \\
& \left.\qquad \begin{array}{l}
\phi \\
\theta \\
\psi
\end{array}\right\}=\left\{\begin{array}{c}
2 \sin ^{-1}\left[\frac{e_{x}}{\cos (\pi / 4)}\right]+\psi \\
\pi / 2 \\
\text { arbitrary }
\end{array}\right\} \\
& \operatorname{elif}\left(e_{0} e_{y}-e_{x} e_{z}=-0.5\right) \quad \\
& \left\{\begin{array}{l}
\phi \\
\theta \\
\psi
\end{array}\right\}=\left\{\begin{array}{c}
2 \sin ^{-1}\left[\frac{e_{x}}{\cos (\pi / 4)}\right]-\psi \\
-\pi / 2 \\
\operatorname{arbitrary}
\end{array}\right\}
\end{aligned}
$$

else

$$
\left\{\begin{array}{l}
\phi \\
\theta \\
\psi
\end{array}\right\}=\left\{\begin{array}{c}
\operatorname{atan} 2\left[2\left(e_{0} e_{x}+e_{y} e_{z}\right),\left(e_{0}^{2}+e_{x}^{2}-e_{y}^{2}-e_{z}^{2}\right)\right] \\
\sin ^{-1}\left[2\left(e_{0} e_{y}-e_{x} e_{z}\right)\right] \\
\operatorname{atan} 2\left[2\left(e_{0} e_{z}+e_{x} e_{y}\right),\left(e_{0}^{2}+e_{x}^{2}-e_{y}^{2}-e_{z}^{2}\right)\right]
\end{array}\right\}
$$

The heading at the gimbal lock conditions is arbitrary, therefore any convenient value may be used during simulation.

\section{Flight Dynamics Model}

The equations of motion used for simulating the aircraft behavior follow Phillip's derivation of a 13 state, firstorder, differential set of equations [7]. This set of equations is based on a local flat-Earth approximation which includes gyroscopic and inertial coupling. The adopted 13-state model is

$$
\begin{aligned}
&\left\{\begin{array}{c}
\dot{u} \\
\dot{v} \\
\dot{w}
\end{array}\right\}= \frac{g}{W}\left\{\begin{array}{l}
F_{x_{b}} \\
F_{y_{b}} \\
F_{z_{b}}
\end{array}\right\}+g\left\{\begin{array}{c}
2\left(e_{x} e_{z}-e_{y} e_{0}\right) \\
2\left(e_{y} e_{z}+e_{x} e_{0}\right) \\
e_{z}^{2}+e_{0}^{2}-e_{x}^{2}-e_{y}^{2}
\end{array}\right\}+\left\{\begin{array}{l}
r v-q w \\
p w-r u \\
q u-p v
\end{array}\right\} \\
&\left\{\begin{array}{l}
\dot{p} \\
\dot{q} \\
\dot{r}
\end{array}\right\}= {\left[\begin{array}{ccc}
I_{x x} & -I_{x y} & -I_{x z} \\
-I_{x y} & I_{y y} & -I_{y z} \\
-I_{x z} & -I_{y z} & I_{z z}
\end{array}\right]^{-1}\left(\begin{array}{ccc}
0 & -h_{z} & h_{y} \\
h_{z} & 0 & -h_{x} \\
-h_{y} & h_{x} & 0
\end{array}\right]\left\{\begin{array}{l}
p \\
q \\
r
\end{array}\right\}+} \\
&\left.\left\{\begin{array}{l}
M_{x_{b}}+\left(I_{y y}-I_{z z}\right) q r+I_{y z}\left(q^{2}-r^{2}\right)+I_{x z} p q-I_{x y} p r \\
M_{y_{b}}+\left(I_{z z}-I_{x x}\right) p r+I_{x z}\left(r^{2}-p^{2}\right)+I_{x y} q r-I_{y z} p q \\
M_{z_{b}}+\left(I_{x x}-I_{y y}\right) p q+I_{x y}\left(p^{2}-q^{2}\right)+I_{y z} p r-I_{x z} q r
\end{array}\right\}\right) \\
&\left.\left\{\begin{array}{l}
\dot{x}_{f} \\
\dot{y}_{f} \\
\dot{z}_{f}
\end{array}\right\}=\left\{\begin{array}{l}
e_{0} \\
e_{x} \\
e_{y} \\
e_{z}
\end{array}\right\} \otimes\left(\begin{array}{l}
0 \\
u \\
v \\
w
\end{array}\right\} \otimes\left\{\begin{array}{c}
e_{0} \\
-e_{x} \\
-e_{y} \\
-e_{z}
\end{array}\right\}\right)
\end{aligned}
$$




$$
\left\{\begin{array}{l}
\dot{e}_{0} \\
\dot{e}_{x} \\
\dot{e}_{y} \\
\dot{e}_{z}
\end{array}\right\}=\frac{1}{2}\left[\begin{array}{ccc}
-e_{x} & -e_{y} & -e_{z} \\
e_{0} & -e_{z} & e_{y} \\
e_{z} & e_{0} & -e_{x} \\
-e_{y} & e_{x} & e_{0}
\end{array}\right]\left\{\begin{array}{l}
p \\
q \\
r
\end{array}\right\}
$$

where $g$ is the gravitational acceleration, $W$ is the weight of the aircraft, $u, v, w$, and $p, q, r$ are the body-fixed translational and rotational velocities in the $x_{b}, y_{b}$, and $z_{b}$ directions, respectively, and $h$ is the angular momentum in the body-fixed coordinate system. Note that Eq. (41) is the transformation of a vector from body-fixed coordinates to Earth-fixed coordinates. These 13 differential equations are integrated forward in the time-domain at a desired timestep using a fourth-order Runge Kutta integration scheme to yield the 13 states of the aircraft at discrete moments in time. All 13 equations are integrated during a single timestep, and the quaternion is renormalized after each timestep to prevent orthogonality error [7]. The 13 states represented in Eq. (39) through Eq. (42) are the states used in the system identification process in Section VI.

\section{Timestep Selection}

The timestep used in the flight simulation for the baseline and trial flights can have a large impact on the accuracy of the predicted flight path in the simulator. Generally, the smaller the timestep of a simulation, the more accurate the results. However, there is a point where the added computational cost and time are not worth the small gains in accuracy from the simulation. Phillips discusses this in [7]. He concludes that the timestep for a fourth-order RungeKutta integration scheme can be as high as 0.08 seconds without significant error [7]. Additionally, Jategaonkar [5] explains that in system identification for rigid-body aerodynamic model estimation, a sampling frequency of $20-25$ $\mathrm{Hz}$ is sufficient, which corresponds to a timestep between $0.05-0.04$ seconds.

Most standard data recorders for prototype SUAS aircraft generally have a sampling rate of $25 \mathrm{~Hz}$. For example, the popular Pixhawk Cube reports the data at $25 \mathrm{~Hz}$ to the user. To avoid linear interpolation of the flight recorder data later, the timestep for simulation was chosen to be 0.04 seconds or $25 \mathrm{~Hz}$. This timestep is right around the maximum timestep Phillips recommends without sacrificing too much fidelity in the simulation. An added benefit of this timestep is that it results in faster run times and lower computational cost.

\section{Aircraft Description and Analysis}

The process and results detailed in this paper correspond to the aircraft described in this section. The baseline aircraft is a rudderless, flying wing with linearly changing sweep and dihedral as shown in Fig. 4. Tables 1-3 describe the aircraft setup and aerodynamic properties used later in the system identification process. The aerodynamic properties in Table 3 were determined using MachUpX. MachUpX has a function that auto-populates the stability, damping, and control derivatives using a finite-difference scheme. In this paper, derivatives were calculated using a grid spacing of 80 control points along each semi-span with a required residual convergence of $1 \mathrm{E}-10$.

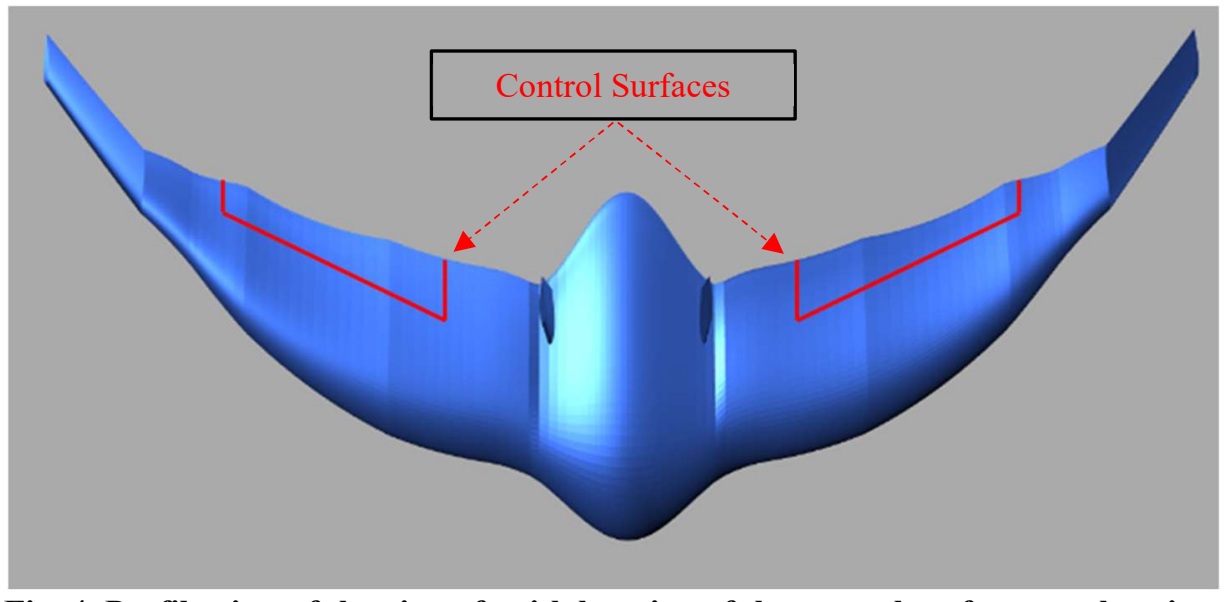

Fig. 4 Profile view of the aircraft with location of the control surfaces on the wing.

The aircraft uses an E-flite Power $32770 \mathrm{Kv}$ brushless motor with a $14.8 \mathrm{~V}$ battery and a $14 \times 6.5$ inch propeller as its propulsion system. The propulsion information in Table 1 was obtained through wind tunnel experimentation and 
through a least-squares fit of the thrust data to the equations for thrust given by Eqs. (31) - (34). The inertia tensor for the aircraft, shown in Table 2, was determined using SolidEdge ${ }^{\S}$ with the propulsion system onboard. The values in Table 3 were all determined at the aircraft reference condition described in Table 1 . The reference condition uses a cruise flight speed at standard sea level atmospheric conditions. The root of the control surfaces (elevons) are located at the $40 \%$ semi-span location with a chord fraction of $35 \%$ of the local chord, while the tip of the elevons are at the $90 \%$ semi-span with a chord fraction of $25 \%$ of the local chord as shown in Fig. 4.

Table 1 Aircraft geometry, reference condition and propulsion system information.

\begin{tabular}{cccccc}
\hline \hline & Geometric & \multicolumn{2}{c}{ Reference Condition } & \multicolumn{2}{c}{ Propulsion } \\
\hline$b_{w}$ & $5.85 \mathrm{ft}$ & $V$ & $69 \mathrm{ft} / \mathrm{sec}$ & $T_{0}$ & 3.8 \\
$\bar{c}_{w}$ & $1.303 \mathrm{ft}$ & $\rho_{0}$ & $2.3769 \mathrm{E}-3 \mathrm{slug} / \mathrm{ft}^{3}$ & $T_{1}$ & -0.03870 \\
$S_{w}$ & $7.62 \mathrm{ft}^{2}$ & $W$ & $12 \mathrm{lbs}$ & $T_{2}$ & 0.00005 \\
& & & $C_{D_{P}}$ & 0.001193 \\
& & & $S_{p}$ & $1 \mathrm{ft}^{2}$ \\
& & & $a$ & 1 \\
\hline \hline
\end{tabular}

Table 2 Aircraft inertia tensor in slug-ft ${ }^{2}$.

\begin{tabular}{cccccc}
\hline \hline$I_{x x}$ & 0.2950 & $I_{x y}$ & 0 & $I_{x z}$ & 0.0096 \\
$I_{y x}$ & 0 & $I_{y y}$ & 0.1430 & $I_{y z}$ & 0 \\
$I_{z x}$ & 0.0096 & $I_{z y}$ & 0 & $I_{z z}$ & 0.4310 \\
\hline \hline
\end{tabular}

Table 3 Aircraft aerodyamic properties.

\begin{tabular}{|c|c|c|c|c|c|c|c|}
\hline \multicolumn{2}{|c|}{ General } & \multicolumn{2}{|c|}{ Stability } & \multicolumn{2}{|c|}{ Damping } & \multicolumn{2}{|c|}{ Control } \\
\hline$C_{L_{0}}$ & 0.06452 & $C_{L, \alpha}$ & 3.56058 & $C_{S, \bar{p}}$ & -0.36794 & $C_{L, \delta_{e}}$ & 0.87480 \\
\hline$C_{m_{0}}$ & 0.01996 & $C_{m, \alpha}$ & -0.62446 & $C_{\ell, \bar{p}}$ & -0.46335 & $C_{D, \delta_{e}}$ & 0.01533 \\
\hline$C_{D 0}$ & 0.01741 & $C_{S, \beta}$ & -0.23098 & $C_{n, \bar{p}}$ & 0.05265 & $C_{m, \delta_{e}}$ & -0.43817 \\
\hline$C_{D 1}$ & -0.04249 & $C_{\ell, \beta}$ & -0.13596 & $C_{L, \bar{q}}$ & 0.88332 & $C_{S, \delta_{a}}$ & -0.14557 \\
\hline$C_{D 2}$ & 0.12852 & $C_{n, \beta}$ & 0.05088 & $C_{D, \bar{q}}$ & -0.06115 & $C_{\ell, \delta_{a}}$ & -0.24816 \\
\hline \multirow[t]{4}{*}{$C_{D 3}$} & 1.54407 & & & $C_{m, \bar{q}}$ & -0.76715 & $C_{n, \delta_{a}}$ & 0.03286 \\
\hline & & & & $C_{S, \bar{r}}$ & 0.08024 & & \\
\hline & & & & $C_{\ell, \bar{r}}$ & 0.04145 & & \\
\hline & & & & $C_{n, \bar{r}}$ & -0.02444 & & \\
\hline
\end{tabular}

\section{Sensitivity Analysis}

This section presents the method used for measuring and analyzing the parameter sensitivity for 33 aircraft parameters. The first part outlines which parameters are evaluated and how sensitivity is measured. The second part describes how certain flight maneuvers were formulated and performed to evaluate the sensitivity of the 33 parameters. A closed form, analytic approximation for aircraft natural frequencies is presented in this section. Lastly, the results of the sensitivity analysis are presented for the final determined flight plan.

\section{A. Sensitivity Scope}

There are 33 changeable flight parameters in the aircraft model, which are listed in the propulsion section of Table 1,2 , and 3 , excluding $a, S_{P}$, and the off-diagonal terms in the inertia tensor. The thrust parameter $a$ was neglected from this analysis because all the flight simulation was conducted at Standard Seal Level (SSL) density, 2.3769E-3 slug/ $\mathrm{ft}^{3}$. The thrust parameter $a$ is a scaling factor that acts on the air density ratio in Eq. (32). However, since the flight simulation was all done at SSL density, and because changes in density between a few hundred feet are nearly negligible for SUAS aircraft of this size, the ratio $\left(\rho / \rho_{0}\right)$ from Eq. (32) is assumed to be unity throughout the simulation. Table 4 outlines the complete list of flight parameters evaluated in this section and estimated in the next section.

\footnotetext{
$\S \underline{\text { https://solidedge.siemens.com/en/ }}$
} 
Table 4 The 33 aerodynamic, propulsion, and inertial parameters being evaluated in this work.

\begin{tabular}{cc|cc}
\hline \hline & Longitudinal & & Lateral \\
\hline$C_{L, \alpha}$ & $C_{D, \bar{q}}$ & $C_{\ell, \beta}$ & $C_{\ell, \bar{r}}$ \\
$C_{m, \alpha}$ & $C_{D 0}$ & $C_{n, \beta}$ & $C_{n, \bar{r}}$ \\
$C_{L_{0}}$ & $C_{S, \beta}$ & $C_{S, \bar{r}}$ \\
$C_{m_{0}}$ & $C_{\ell, \delta_{a}}$ & $C_{D 3}$ \\
$C_{L, \delta_{e}}$ & $C_{n, \delta_{a}}$ & $I_{x x}$ \\
$C_{m, \delta_{e}}$ & $C_{S, \delta_{a}}$ & $I_{z z}$ \\
$C_{D, \delta_{e}}$ & $C_{D 2}$ & \\
$C_{L, \bar{q}}$ & $T_{0}$ & $C_{\ell, \bar{p}}$ & \\
$C_{m, \bar{q}}$ & $T_{1}$ & $C_{n, \bar{p}}$ & \\
\hline \hline
\end{tabular}

Two separate aircraft will be referred to throughout this and the following section on sensitivity and parameter estimation respectively. These two aircraft are the baseline aircraft and the trial aircraft. Evaluating the difference between the baseline states and the trial states from the simulator allows us to understand how sensitive the flight plan is to a given flight parameter. The objective of this analysis is to determine the longitudinal and lateral flight maneuvers necessary to accurately estimate the aircraft parameters in the following section. This section outlines the analysis and results of the sensitivity of flight characteristics to the flight parameters. Sensitivity, in this document, refers to the measure of how responsive the aircraft state file from the simulator is to any of the 33 aircraft parameters listed in Table 4.

\section{Quantifying Parameter Sensitivity}

The sensitivity of a flight to a set of parameters was determined with two measurement schemes. Initially, the sensitivity was measured by evaluating the difference between all of the baseline and trial states. The sensitivity was recorded as the $R M S E_{a}$, which is calculated by

$$
\operatorname{RMSE}_{a}=\sqrt{\sum_{i=1}^{\eta} \sum_{j=1}^{13} \frac{\left(\tilde{s}_{i j}-s_{i j}\right)^{2}}{\eta \times 13}}
$$

where $\eta$, is the number of timesteps in the state files, $\tilde{s}_{i j}$, is the baseline states, and $s_{i j}$ is the trial states. This is possible since both the baseline and trial flights are conducted with the same initial conditions, and both use the same control inputs and timesteps for the duration of the flight.

One of the goals of this approach is to implement the method to actual flight data from a prototype aircraft. However, extracting all 13 states from a flight recorder, as opposed to simply running it through the simulator, for an arbitrary prototype, is not a trivial task. The four quaternion states, as well as the body fixed velocities can prove to be difficult to back out of the flight recorder data. As a result, another measurement scheme was used. This second scheme evaluates only the difference between the $x_{f}, y_{f}$, and $z_{f}$ points of the baseline and trial states. This sensitivity measurement method is quantified using the $R M S E_{f}$ calculated by

$$
R M S E_{f}=\sqrt{\sum_{i=1}^{\eta} \sum_{j=7}^{9} \frac{\left(\tilde{s}_{i j}-s_{i j}\right)^{2}}{\eta \times 3}}
$$

where $\eta$ is the number of timesteps in the state files, $\tilde{s}_{i j}$ is the Earth-fixed coordinates for the baseline aircraft, and $s_{i j}$ is the Earth-fixed coordinates for the trial aircraft. Here, $s_{i j}$ is only the Earth-fixed coordinates because $j=7$ to 9 represents the $7^{\text {th }}, 8^{\text {th }}$, and $9^{\text {th }}$ states given in Eq. (41). This scheme was employed since the $x_{f}, y_{f}$, and $z_{f}$ points are easily obtainable from GPS flight test data on a prototype aircraft. The result from Eq. (44) offers a different way to measure the sensitivity at smaller computational cost than Eq. (43). Using this method, the study tells us how sensitive a flight is to a given parameter if all we know are the $x_{f}, y_{f}$, and $z_{f}$ points. Visually, the $R M S E_{f}$ value for a flight can be depicted by Fig. 5 . 


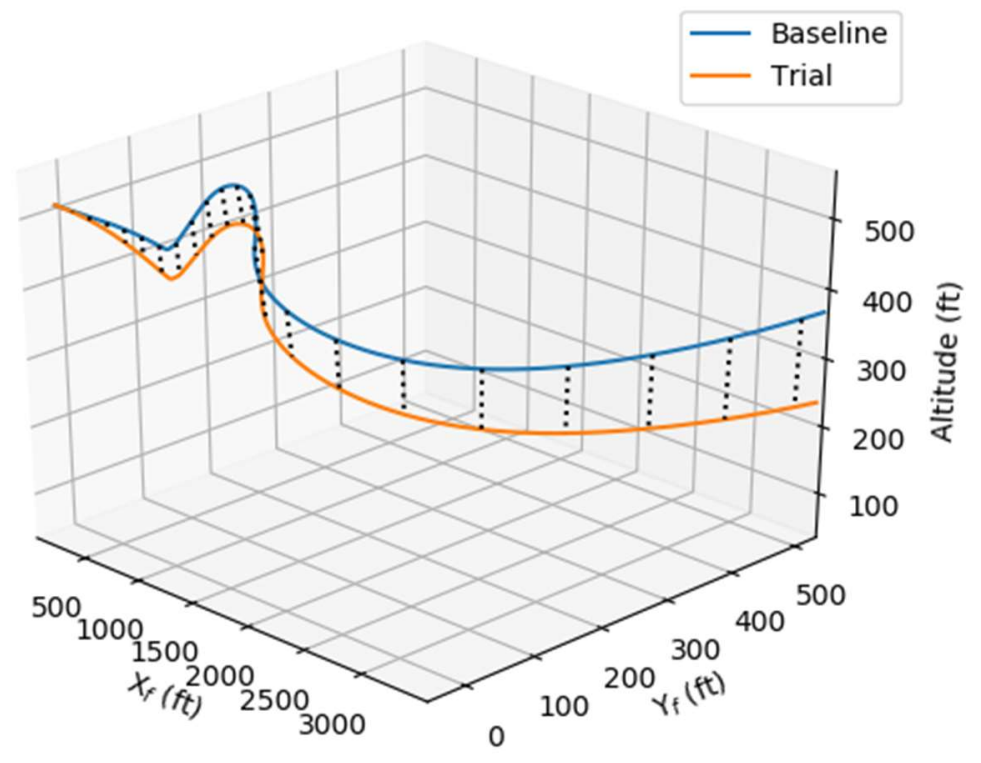

Fig. 5 Comparison of an example flight path of the baseline aircraft and the trial aircraft. The dotted lines represent the $R M S E_{f}$ of the trial aircraft at 2 second intervals in the flight. The trial aircraft has a $1 \%$ change in $C_{L_{0}}$ from the baseline aircraft.

Figure 5 visually represents the $R M S E_{f}$ measurement between the two aircraft at discrete time intervals. However, the $R M S E_{f}$ reported in Eq. (44) is actually a measure of the entire flight $R M S E_{f}$. It is important to recognize that the magnitude of the $R M S E_{f}$ value increases proportionally with respect to the length of the flight. The consequence of this phenomenon is handled in a later section.

\section{B. Flight Testing Maneuvers for System Identification}

The topic of flight test maneuver optimization is a broad and extensive subject that consists of determining the correct maneuvers for flight testing and system identification. This work does not dive into the intricacies of maneuver selection. However, a general explanation of the maneuvers used in this work is presented here. The aim of this section is to outline the chosen maneuvers and describe how they were performed. More detail about flight testing and its nuances can be found in [19-21]. Some of the maneuvers analyzed in this work require a timestep for control inputs in order to excite specific modes in the aircraft. There are different methods for determining an adequate timestep for acceptable mode excitation [2, 5, 22]. Before describing the maneuvers used in this study, a brief overview of the timestep determination is outlined. As part of the discussion on the timestep, a closed form solution for predicting the natural frequencies of certain modes is presented. The natural frequency is used to derive the timestep for certain maneuvers.

\section{Closed Form Approximations for Aircraft Natural Frequencies}

Phillips [7] presents a closed-form method for approximating the natural frequencies of the short period, phugoid, and Dutch roll modes. These approximations were used to estimate the natural frequencies needed for determining the timestep for specific control inputs described in the next section. His equations are expressed in terms of dimensionless parameters given by

$$
\begin{array}{cc}
R_{z, \alpha}=\frac{\rho S_{w} \bar{c}_{w}}{4 W / g}\left(-C_{L, \alpha}-C_{D o}\right) & R_{m, \bar{q}}=\frac{\rho S_{w} \bar{c}_{w}^{3}}{8 I_{y y}} C_{m, \bar{q}} \\
R_{m, \widehat{\alpha}}=\frac{\rho S_{w} \bar{c}_{w}}{8 I_{y y}} C_{m, \widehat{\alpha}} & R_{m, \alpha}=\frac{\rho S_{w} \bar{c}_{w}^{3}}{8 I_{y y}} C_{m, \alpha} \\
R_{x, \mu}=\frac{\rho S_{w} \bar{c}_{w}}{4 W / g}\left(-2 C_{D o}\right) & R_{z, \mu}=\frac{\rho S_{w} \bar{c}_{w}}{4 W / g}\left(-2 C_{L o}\right)
\end{array}
$$




$$
\begin{array}{cc}
R_{x, \alpha}=\frac{\rho S_{w} \bar{c}_{w}}{4 W / g}\left(C_{L o}-C_{D, \alpha}\right) & R_{s}=\frac{R_{m, \alpha}}{R_{m, \alpha}-R_{z, \alpha} R_{m, \bar{q}}} \\
R_{d}=\frac{R_{x, \alpha} R_{m, \bar{q}}}{R_{m, \alpha}-R_{z, \alpha} R_{m, \bar{q}}} & R_{y, \bar{r}}=\frac{\rho S_{w} b_{w}}{4 W / g} C_{y, \bar{r}} \\
R_{n, \bar{r}}=\frac{\rho S_{w} b_{w}^{3}}{8 I_{z z}} C_{n, \bar{r}} & R_{\ell, \bar{r}}=\frac{\rho S_{w} b_{w}^{3}}{8 I_{x x}} C_{\ell, \bar{r}} \\
R_{\ell, \beta}=\frac{\rho S_{w} b_{w}^{3}}{8 I_{x x}} C_{\ell, \beta} & R_{n, \beta}=\frac{\rho S_{w} b_{w}^{3}}{8 I_{z z}} C_{n, \beta} \\
R_{y, \beta}=\frac{\rho S_{w} b_{w}}{4 W / g} C_{y, \beta} & R_{n, \bar{p}}=\frac{\rho S_{w} b_{w}^{3}}{8 I_{z z}} C_{n, \bar{p}} \\
R_{\ell, \bar{p}}=\frac{\rho S_{w} b_{w}^{3}}{8 I_{x x}} C_{\ell, \bar{p}} & R_{g y}=\frac{g b_{w}}{2 V_{0}^{2}} \\
R_{g x}=\frac{g \bar{c}_{w}}{2 V_{0}^{2}} & R_{D s}=\frac{R_{\ell, \beta}\left[R_{g y}-\left(1-R_{y, \bar{r}}\right) R_{n, \bar{p}}\right]-R_{y, \beta} R_{\ell, \bar{r}} R_{n, \bar{p}}}{R_{\ell, \bar{p}}}
\end{array}
$$

Each of these dimensionless parameters can be computed from the aircraft data listed in Table 1 - Table 3. In these equations, $V_{0}$ is the reference velocity. The reader should note that $C_{L o}$ is not the same as $C_{L_{0}}$. The first is the lift coefficient at the reference condition in Table 1, and the second is the lift coefficient at zero angle of attack, zero sideslip angle, zero rotational rates, zero translational accelerations, and zero control surface deflection. Similarly, $C_{D o}$ and $C_{D 0}$ are not the same. The first is the drag coefficient at the reference condition and the second is the first drag coefficient in the parabolic equation for the total drag coefficient from Eq. (25). From these dimensionless parameters, Phillips defines the short period damped natural frequency as

$$
\omega_{d_{s p}}=\frac{2 V_{0}}{\bar{c}_{w}} \sqrt{\left(R_{z, \alpha} R_{m, \bar{q}}-R_{m, \alpha}\right)-\left(\frac{R_{z, \alpha}+R_{m, \bar{q}}+R_{m, \widehat{\alpha}}}{2}\right)^{2}}
$$

the phugoid damped natural frequency as

$$
\omega_{d_{p}}=\frac{R_{z, \mu} V_{0}}{\bar{c}_{w}} \sqrt{-\frac{4 R_{g x}}{R_{z, \mu}} R_{s}-\left(\frac{R_{x, \mu}}{R_{z, \mu}}+R_{d}\right)^{2}}
$$

and the Dutch roll damped natural frequency as

$$
\omega_{d d r}=\frac{2 V_{0}}{b_{w}} \sqrt{\left(1-R_{y, \bar{r}}\right) R_{n, \beta}+R_{y, \beta} R_{n, \bar{r}}+R_{D s}-\left(\frac{R_{y, \beta}+R_{n, \bar{r}}}{2}\right)^{2}}
$$

From these approximations the short period damped natural frequency of the aircraft is $14.48 s^{-1}$, the phugoid damped natural frequency is $0.59 s^{-1}$, and the Dutch roll damped natural frequency is $4.58 s^{-1}$. These frequencies will be used to determine the timestep of the control inputs for doublets and other multi-step inputs. 


\section{Pulse, Doublet, and Multi-Step Inputs}

There are multiple different input patterns that can be applied to an aircraft to excite oscillatory motion. Various texts present different ways of determining the control input timesteps for a given maneuver [2,5]. Each input pattern attempts to excite specific modes to the point that the parameters responsible for a particular mode are identifiable in the data. The simplest control input is known as the pulse input and is performed by applying an input over a specified time period, then the controls are released, and the aircraft is allowed to freely oscillate. The major limitation to this input is that it struggles to excite the rapid short period mode.

The doublet input is more common in aircraft testing and system identification. This control input is performed by rapidly applying a control input in one direction, holding the input for a given timestep, $\Delta t_{D B L T}$, then moving abruptly to the other direction where the control is held fixed for the same specified timestep before the control is returned to neutral [5]. Different authors present different methods for calculating the timestep for the doublet. Jategaonkar [5] calculates the doublet timestep as

$$
\Delta t_{D B L T} \approx \frac{2.3}{\omega_{n}}
$$

Plugging in the values for the natural frequencies from the previous section into Eq. (49) means that $\Delta t_{D B L T}=$ 0.16 seconds for the short period, $\Delta t_{D B L T}=3.88$ seconds for the phugoid, and $\Delta t_{D B L T}=0.5$ seconds for the Dutch roll. The doublet input is widely used for estimation of stability and control derivatives due to its simplicity.

The 3-2-1-1 is a popular input designed by Koehler to increase the energy spectrum over a larger range of input frequencies [23]. The 3-2-1-1 spans seven timesteps and is performed by alternating positive and negative inputs of equal amplitude for three, two, one, and then one timesteps. For example, a 3-2-1-1 elevator input might look like a positive elevator deflection for three timesteps, immediately followed by a negative elevator deflection of the same magnitude for two timesteps, followed by positive elevator deflection of the same magnitude for one timestep. The maneuver is finished with a final negative elevator deflection of the same magnitude for one timestep and the elevator is relaxed while the aircraft is allowed to oscillate. Jategaonkar [5] computes the timestep for the 3-2-1-1 input by

$$
\Delta t_{3211} \approx \frac{1.6}{\omega_{n}} \text { or } \Delta t_{3211} \approx \frac{2.1}{\omega_{n}}
$$

In this work, we took the average of the two equations for one timestep calculation by

$$
\Delta t_{3211} \approx \frac{1.85}{\omega_{n}}
$$

From this equation, the $\Delta t_{3211}=0.13$ seconds for the short period, $\Delta t_{3211}=3.12$ seconds for the phugoid, and $\Delta t_{3211}=0.40$ seconds for the Dutch roll.

Alternatively, Morelli [22] determines $\Delta t_{3211}$ by matching a single pulse in the 3-2-1-1 to one-half the period of the desired oscillatory mode. Since the period is related to the natural frequency by

$$
\mathrm{T}_{m}=\frac{2 \pi}{\omega_{n}}
$$

then Morellis calculation of $\Delta t_{3211}$ gives

$$
\Delta t_{3211} \approx \frac{2 \pi}{2 \omega_{n}}=\frac{\pi}{\omega_{n}}=\frac{\mathrm{T}_{m}}{2}
$$

From Morelli's method, $\Delta t_{3211}=0.21$ seconds for the short period, $\Delta t_{3211}=5.30$ seconds for the phugoid, and $\Delta t_{3211}=0.69$ seconds for the Dutch roll. Both, Jategaonkar's [5] and Morelli's [22] methods were used in different Dutch roll and short period flights to see if the difference in timestep would help identify certain parameters better. The difference between the results produced by the two timesteps was negligible. 


\section{Description of the Maneuvers Analyzed}

The following is the list of maneuvers analyzed:

- Acceleration-Deceleration

- Bank-to-Bank

- Barrel Roll

- Climbing Turns

- Dutch Roll - Pulses

- Dutch Roll Doublet/3-2-1-1 $\left(\Delta t_{3211}=0.40\right)$

- Dutch Roll Doublet/3-2-1-1 $\left(\Delta t_{3211}=0.69\right)$

- Level Turn with Elevator Doublet

- Phugoid (10 second elevator pulse)

- Phugoid (thrust variation)

- Pushover-Pullups

- Rapid Throttle Changes

- $\quad$ Short Period 3-2-1-1 $\left(\Delta t_{3211}=0.13\right)$

- $\quad$ Short Period 3-2-1-1 $\left(\Delta t_{3211}=0.21\right)$

- Slow Throttle Changes

- Spiral

A brief description of how each maneuver is performed is given for context. Each of the maneuvers starts at the trimmed level flight condition represented in Table 1.

The acceleration-deceleration maneuver is performed by reducing the power to idle and maintaining altitude using the elevator. Once the speed $20 \%$ greater than stall is reached, full power is applied, accelerating the aircraft. Altitude is maintained through elevator deflection until the maximum speed is reached, then power is returned to idle until the aircraft is returned to the initial trim speed, at which point the maneuver is terminated.

The bank-to-bank maneuver is performed with a series of aileron inputs. Again, starting from level trimmed flight, a rapid aileron input is applied and held for enough time to roll the aircraft to at least $30^{\circ}$ bank. This is immediately followed by a rapid reversal of the aileron input and smooth roll back through a wings-level configuration to the equal and opposite bank angle. The maneuver is terminated by returning to wings-level. This test is repeated for a variety of bank angles. The maneuver used in this document was performed at $30^{\circ}$ and $60^{\circ}$ of bank.

The barrel roll maneuver is performed by lowering the nose to gain some airspeed, raising the nose about $5^{\circ}$ above the horizon, and rapidly rolling the aircraft $360^{\circ}$ around it's longitudinal axis with a sharp aileron input. This is done in both roll directions.

Climbing turns are performed by increasing the throttle in order to maintain the climb in a turn, rolling to roughly a $20^{\circ}$ bank, and adding enough elevator input to maintain a gradual climb through the turn. Two turns in each direction are completed at roughly the same bank angle and climb rate. The maneuver is terminated at the end of the fourth turn with the wings brought back to level.

The Dutch roll can be a difficult mode to excite without a rudder. In this work, the Dutch roll mode is excited with aileron pulses and doublets and then the aircraft is allowed to oscillate through several cycles of oscillation.

The level turn is performed by rolling the aircraft to $30^{\circ}, 45^{\circ}$, or $60^{\circ}$ bank and holding the bank attitude constant for several seconds. The aircraft is then rolled in the opposite direction to the desired bank angle and the attitude is held constant for several seconds. The maneuver is completed by returning the aircraft to a wings-level configuration. Altitude should be maintained in this maneuver by adding power. The maneuver is typically combined with an elevator doublet during the banked portion of the maneuver. This is done in an attempt to separate the rate of change of angle of attack and the pitch rate components from the longitudinal motion [5].

The phugoid may be excited in multiple ways. One way is to input a small constant elevator input for about 10 seconds, then let go of the controls and let the aircraft oscillate through a few periods of oscillation. Another common way of exciting the phugoid mode is with a thrust variation. This can be done by increasing or decreasing the throttle from the trim condition for a couple seconds and then returning to trim power setting and allowing the aircraft to oscillate through a few oscillation periods.

The pushover-pullup maneuver is primarily used to determine the lift and drag characteristics and longitudinal stability parameters. This maneuver is performed by slowly pushing the nose of the aircraft down until the maximum speed of the aircraft is reached. Then the nose of the aircraft is slowly brought back up, which leads to a decelerating climb. Once the minimum speed (generally $10 \%$ above the stall speed) is reached, the nose of the aircraft is lowered 
once again. This process is repeated a few times and then the aircraft is returned to the starting trimmed condition. The aircraft's throttle is held constant through the entire maneuver.

A rapid throttle change is performed in order to determine the thrust characteristics of the aircraft. The maneuver is performed by rapidly increasing the throttle to full throttle, waiting a couple of seconds, then rapidly changing to idle and waiting for a couple seconds. The process is repeated a few times, and the aircraft is brought back to the starting trimmed throttle setting.

The short-period mode is typically excited with rapid elevator inputs. This is usually done with an elevator doublet, or another multi-step input, such as the 3-2-1-1 input. Slow throttle changes are performed to determine the thrust characteristics. This maneuver is similar to the rapid throttle change. The only major difference between the two is the speed at which the throttle is changed. The process of slowly alternating between full throttle and idle is repeated a few times, and the aircraft is returned to the starting trim setting.

Finally, the spiral mode is a lateral mode characterized by an aircraft's ability to track a given heading and direction of travel through the air [7]. This mode is excited by starting from a trimmed level flight condition. The throttle is brought to idle and a lateral disturbance is introduced, typically with a small but rapid aileron input. The aircraft is allowed to travel in its new course without pilot input correction. If the aircraft enters an increasingly tighter, diving spiral, the mode is said to be divergent.

\section{Sensitivity Methods}

The sensitivity analysis starts by flying the baseline aircraft through a given maneuver, or series of maneuvers, and recording the control inputs and states for the flight. The aircraft is flown through the Pylot ${ }^{* *}$ simulator, which uses the aerodynamic model and equations of motion outlined in previous sections. Pylot is a python based flight simulator. Then, each trial aircraft parameter is individually varied by $1 \%$ from the baseline parameters listed in Table 3 . Varying one parameter at a time, the trial aircraft is run through the simulation with the same control inputs as the baseline flight, and the states of the trial aircraft are recorded for comparison. The resulting $R M S E_{f}$ values are calculated and recorded. This is done for each of the 33 parameters for a given set of flight maneuvers. For each maneuver, different parameters will affect the state of the aircraft and the $R M S E_{f}$ differently. For example, looking at a flight with strictly longitudinal maneuvers, such as an elevator 3-2-1-1 input, the longitudinal parameters $C_{m, \alpha}$, and $C_{L, \alpha}$ have larger $R M S E_{f}$ values than the lateral parameters. This can be seen in Fig. 6.

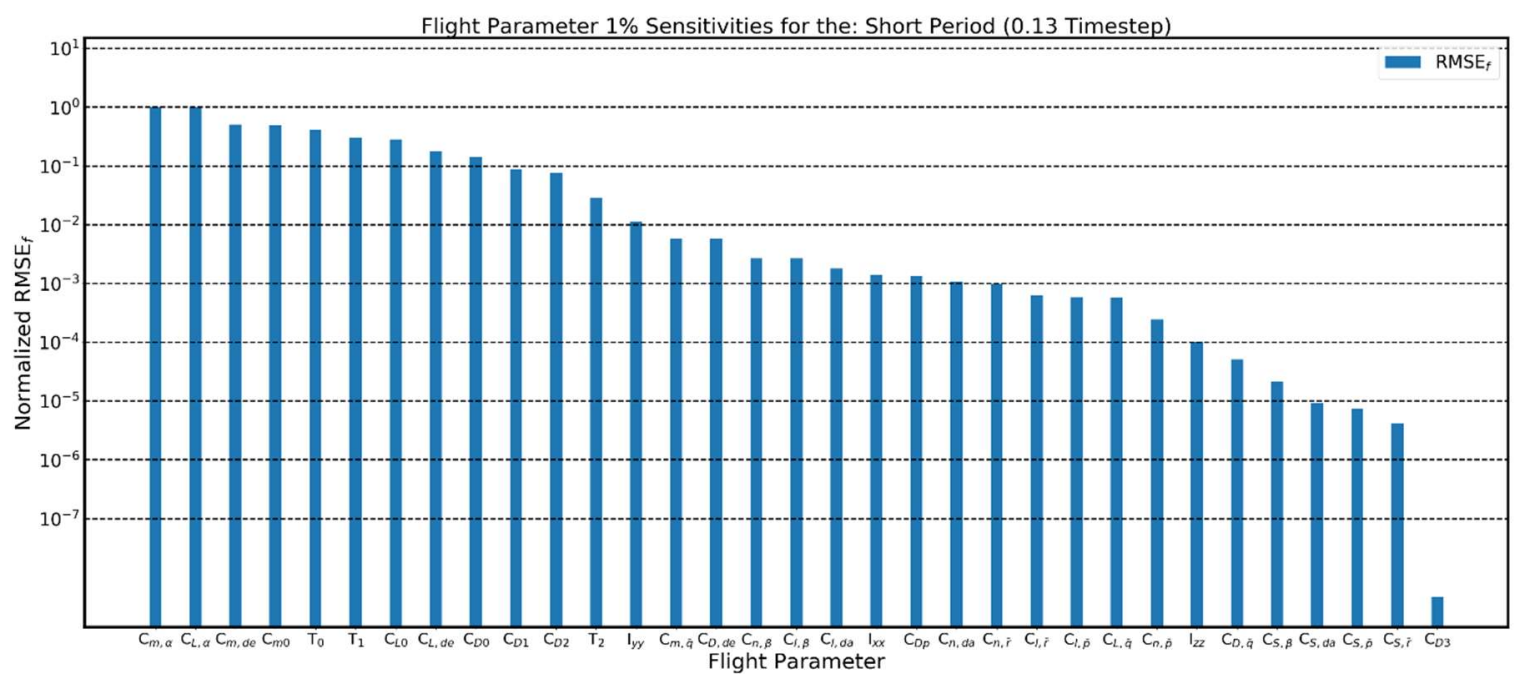

Fig. 6 Normalized $R M S E_{f}$ for each parameter during a 3-2-1-1 elevator maneuver plotted on a logarithmic $y$ axis. Each parameter is normalized by the parameter with the largest $R M S E_{f}$. The values can be found in Table A.1

In order to have a more uniform comparison between each flight, the $R M S E_{f}$ values of each flight are normalized by the largest parameter $R M S E_{f}$. This is done because longer flights have larger $R M S E_{f}$ values since the error grows with time in the simulation.

${ }^{* *}$ https://github.com/usuaero/Pylot 
By comparison, a lateral flight maneuver would have much higher values for the lateral parameters. Figure 7 shows the normalized results for the parameters during a lateral maneuver.

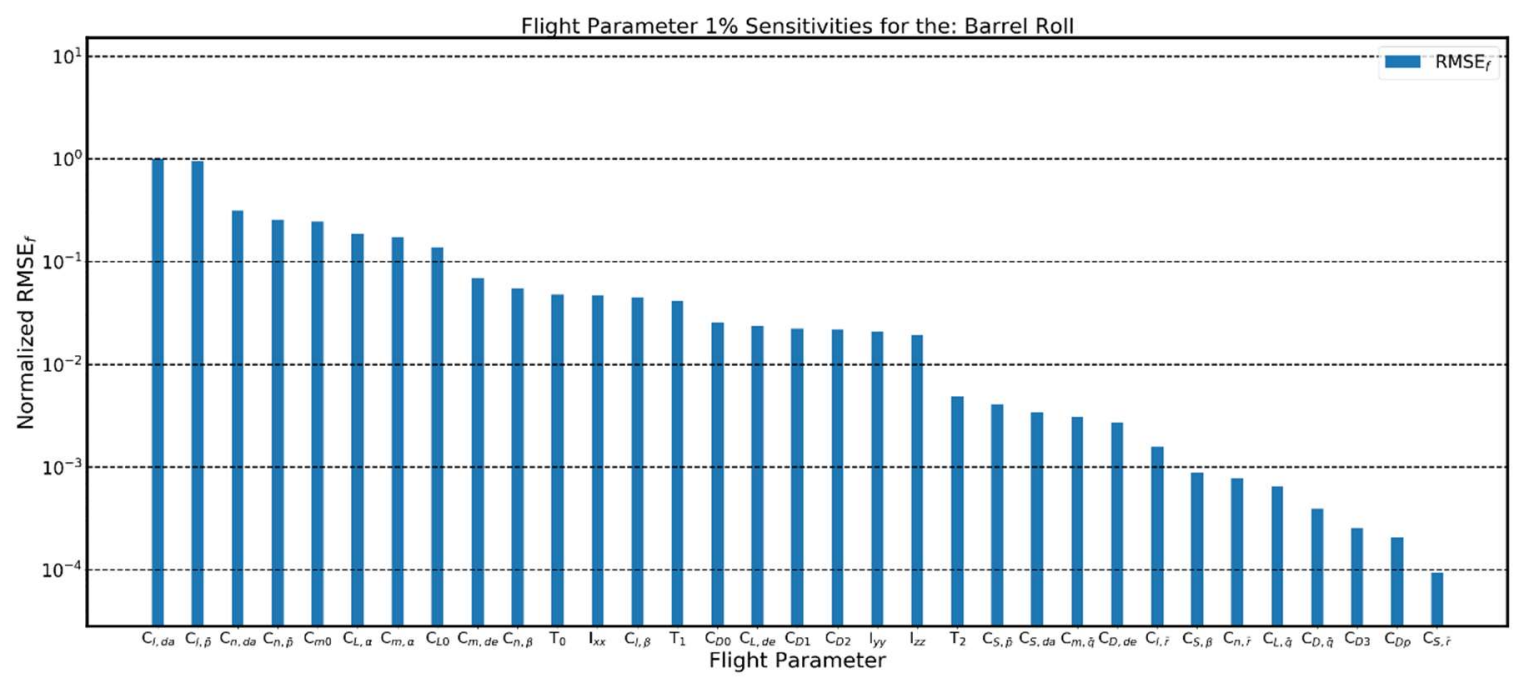

Fig. 7 Normalized $R M S E_{f}$ for each parameter during a lateral flight maneuver plotted on a logarithmic $y$ axis. Each parameter is normalized by the parameter with the largest $R M S E_{f}$. The values can be found in Table A.2

Here we can see that while we are measuring how sensitive a particular flight is to a given parameter with the $R M S E_{f}$, we can also begin to make observations about which flights have the best chance at identifying specific parameters. For example, from the previous two cases, if the goal was to accurately identify $C_{L, \alpha}$, then an elevator 32-1-1 maneuver would likely be a candidate as a maneuver to perform. Similarly, if we wanted to identify $C_{\ell, \delta a}$ and $C_{\ell, \bar{p}}$, then a barrel roll might be a suitable maneuver.

\section{Maneuver Selection}

The previous process was conducted for the 16 different flight maneuvers previously described. The results from those 16 different maneuvers are summarized in Table 5.

Table 5 Results from the sensitivity study for all the parameters from the 16 different flight maneuvers. The normalized $R M S E_{f}$ value reported in the last column corresponds with the selected manuever for a given parameter.

\begin{tabular}{cccc}
\hline \hline Parameter & $\begin{array}{c}\text { Maneuver with Highest } \\
\text { Norm. } R M S E_{f}\end{array}$ & Selected Maneuver & $\begin{array}{c}\text { Normalized } \\
R M S E_{f}\end{array}$ \\
\hline$C_{m, \alpha}$ & Multiple & Acceleration-Deceleration & 1 \\
$C_{\ell, \delta_{a}}$ & Barrel Roll & Barrel Roll & 1 \\
$C_{\ell, \beta}$ & Spiral & Spiral & 1 \\
$C_{\ell, \bar{p}}$ & Dutch Roll $\Delta_{t}=0.5$ & Dutch Roll $\Delta_{t}=0.5$ & 1 \\
$C_{n, \beta}$ & Spiral & Spiral & 0.9624 \\
$C_{L, \alpha}$ & Multiple & Bank-to-Bank & 0.9559 \\
$C_{n, \bar{r}}$ & Spiral & Spiral & 0.8169 \\
$C_{m, \delta_{e}}$ & Climbing Turns & Climbing Turns & 0.8093 \\
$T_{1}$ & Climbing Turns & Climbing Turns & 0.5907 \\
$C_{m_{0}}$ & Slow Throttle & Level Turn w/ Elevator Doublet & 0.5802 \\
$C_{\ell, \bar{r}}$ & Dutch Roll $\Delta_{t}=0.5$ & Dutch Roll $\Delta_{t}=0.5$ & 0.5237 \\
$T_{0}$ & Climbing Turns & Short Period $\Delta_{t}=0.13$ & 0.4159
\end{tabular}




$\begin{array}{cccc}I_{z z} & \text { Dutch Roll }- \text { Impulses } & \text { Dutch Roll }- \text { Impulses } & 0.3773 \\ C_{n, \delta_{a}} & \text { Dutch Roll }- \text { Impulses } & \text { Dutch Roll }- \text { Impulses } & 0.3636 \\ C_{D 0} & \text { Dutch Roll } \Delta_{t}=0.5 & \text { Dutch Roll } \Delta_{t}=0.5 & 0.3523 \\ C_{L_{0}} & \text { Slow Throttle } & \text { Level Turn w/ Elevator Doublet } & 0.3374 \\ C_{n, \bar{p}} & \text { Dutch Roll } \Delta_{t}=0.5 & \text { Dutch Roll } \Delta_{t}=0.5 & 0.3263 \\ C_{D 2} & \text { Climbing Turns } & \text { Climbing Turns } & 0.2465 \\ C_{L, \delta_{e}} & \text { Pushover-Pullups } & \text { Pushover-Pullups } & 0.2329 \\ C_{D 1} & \text { Dutch Roll } \Delta_{t}=0.5 & \text { Dutch Roll } \Delta_{t}=0.5 & 0.2068 \\ I_{y y} & \text { Dutch Roll }- \text { Impulses } & \text { Dutch Roll }- \text { Impulses } & 0.1318 \\ I_{x x} & \text { Dutch Roll }- \text { Impulses } & \text { Dutch Roll }- \text { Impulses } & 0.0727 \\ C_{m, \bar{q}} & \text { Dutch Roll }- \text { Impulses } & \text { Dutch Roll }- \text { Impulses } & 0.0727 \\ T_{2} & \text { Dutch Roll } \Delta_{t}=0.5 & \text { Dutch Roll } \Delta_{t}=0.5 & 0.0632 \\ C_{S, \beta} & \text { Dutch Roll }- \text { Impulses } & \text { Dutch Roll }- \text { Impulses } & 0.0409 \\ C_{D, \delta_{e}} & \text { Climbing Turns } & \text { Climbing Turns } & 0.0186 \\ C_{S, \delta_{a}} & \text { Dutch Roll }- \text { Impulses } & \text { Dutch Roll }- \text { Impulses } & 0.0136 \\ C_{L, \bar{q}} & \text { Dutch Roll } \Delta_{t}=0.5 & \text { Dutch Roll } \Delta_{t}=0.5 & 0.0103 \\ C_{S, \bar{p}} & \text { Dutch Roll } \Delta_{t}=0.5 & \text { Dutch Roll } \Delta_{t}=0.5 & 0.0095 \\ C_{S, \bar{r}} & \text { Climbing Turns } & \text { Climbing Turns } & 0.0093 \\ C_{D 3} & \text { Climbing Turns } & \text { Climbing Turns } & 0.0093 \\ C_{D P} & \text { Climbing Turns } & \text { Climbing Turns } & 0.0093 \\ C_{D, \bar{q}} & \text { Climbing Turns } & \text { Climbing Turns } & 0.0093\end{array}$

From these results, a flight plan of specific maneuvers was created in order to increase the likelihood of accurately estimating each of the 33 flight parameters. Some of the parameters were not very sensitive to any of the maneuvers, such as $C_{D 3}$ and $C_{D_{p}}$. In the cases where the highest $R M S E_{f}$ achieved for a parameter fell below $10 \%$, the maneuver that yielded the highest normalized $R M S E_{f}$ value for that parameter was selected for the flight plan. Jategaonkar recommends, as a rule of thumb, that a parameter is identifiable if its sensitivity has a magnitude of at least $10 \%$ of the largest parameter's sensitivity [5]. In other words, if the term of interest has a sensitivity within one order of magnitude from the largest sensitivity from that flight, then the parameter should be able to be estimated. The purpose of selecting flight maneuvers is then to create a flight plan that increases as many parameters as possible to within one order of magnitude of the largest parameter sensitivity. The maneuvers needed are combined into one flight plan of the consecutive maneuvers. Between each maneuver, the aircraft is returned to a trimmed level flight condition. The order of maneuvers for the final flight plan is:

1. Climbing Turns

2. Bank-to-Bank

3. Dutch Roll - Pulses

4. Dutch Roll Doublet $/ 3-2-1-1\left(\Delta t_{3211}=0.69\right)$

5. Short Period 3-2-1-1 $\left(\Delta t_{3211}=0.13\right)$

6. Pushover-Pullups

7. Level Turn with Elevator Doublet

8. Barrel Roll

9. Acceleration-Deceleration

10. Spiral

The order of the maneuvers was selected to place the maneuvers responsible for identifying the more difficult parameters towards the beginning of the flight. This was done to influence the entire flight path with disturbances in the less sensitive parameters. Additionally, lateral maneuvers were interspersed throughout the flight to try and keep the entire flight dependent on lateral parameters. 


\section{Final Flight Plan Results}

The final flight plan determined in the previous section was run through the same sensitivity analysis to determine which parameters would be identifiable during parameter estimation. The 3-D graph in Fig. 8 shows the flight path in Earth-fixed space. The flight is color coded when each maneuver is performed during the flight starting with the climbing turns and ending with the spiral. The real-time duration of the flight is 789 seconds, or 13 minutes long.
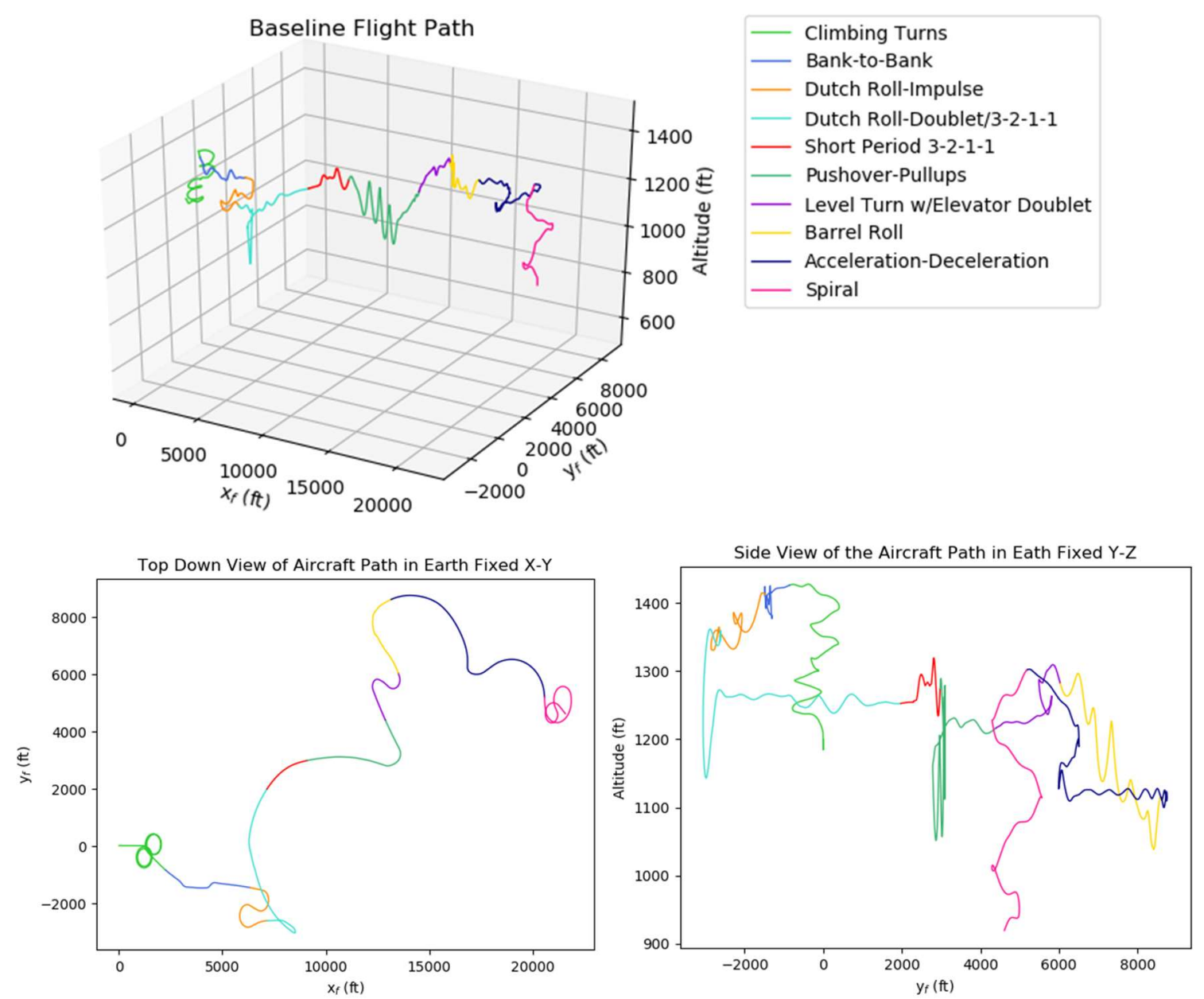

Fig. 8 Top) Final flight plan represented in the Earth-fixed coordinate system with the location of each maneuver color coded. Bottom Left) Top down view of the final flight plan. Bottom Right) Side view of the final flight plan looking along the $x_{f}$ axis.

The control inputs as a function of time for the final flight plan are shown in Fig. 9. Step inputs were used to excite the different modes of the aircraft. For example, Fig. 9 shows that when large aileron inputs are used, the sideslip angle increases during the corresponding maneuvers indicated by the color bar at the bottom of each plot. 

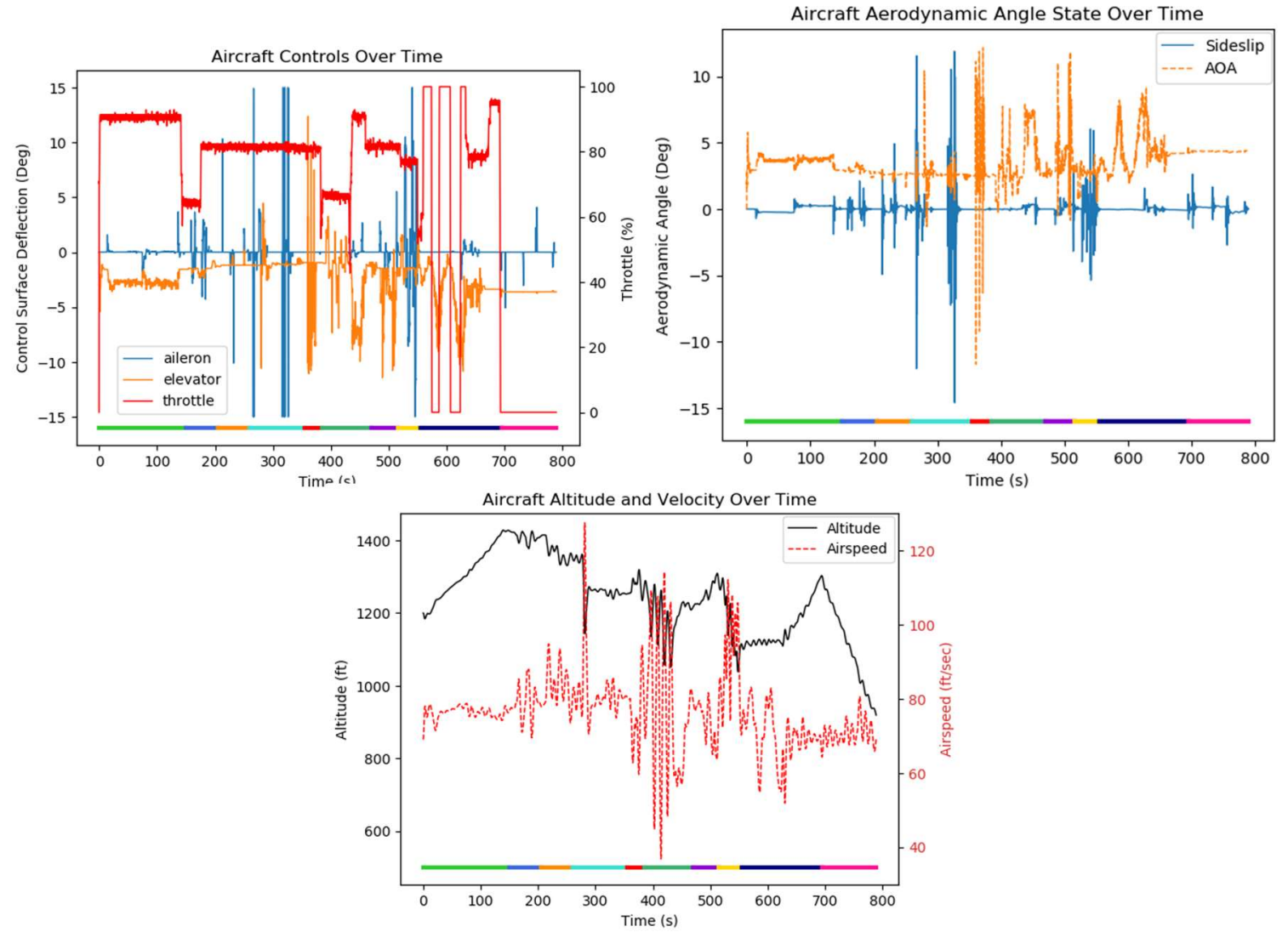

Fig. 9 Top Left) Aircraft controls over the duration of the flight, with aileron and elevator on the left axis and throttle on the right axis. Top Right) Aerodynamic angles over the duration of the flight. Bottom Center) Altitude and airspeed of the aircraft during the flight, with altitude on the left axis and airspeed on the right axis. The color bar on each plot follows the color pattern legend for the maneuver shown in Fig. 8 above.

Figure 10 is a visual representation of what the $R M S E_{f}$ is for the different flight paths for the baseline and trial aircraft with a $1 \%$ difference in $C_{L, \alpha}$. The dotted lines between the baseline and the trial flight paths represent the $R M S E_{f}$ values at 5 second intervals during the flight. The final reported $R M S E_{f}$ for a given trial flight is the average of these $R M S E_{f}$ at each timestep during the flight.

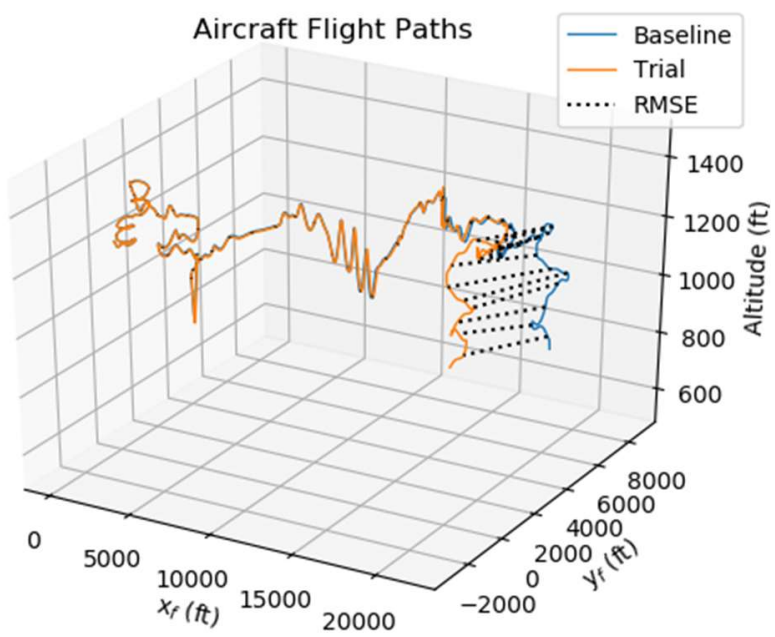

Fig. 10 Differences in flight path from the baseline flight to the trial with a $1 \%$ change in $C_{L, \alpha}$ with the $R M S E_{f}$ value at 5 second intervals marked by dotted lines. 
Lastly, Fig. 11 shows the results of the parameter sensitivity study for all 33 flight parameters during the final flight. The results shown in Fig. 11 are displayed on a logarithmic scale.

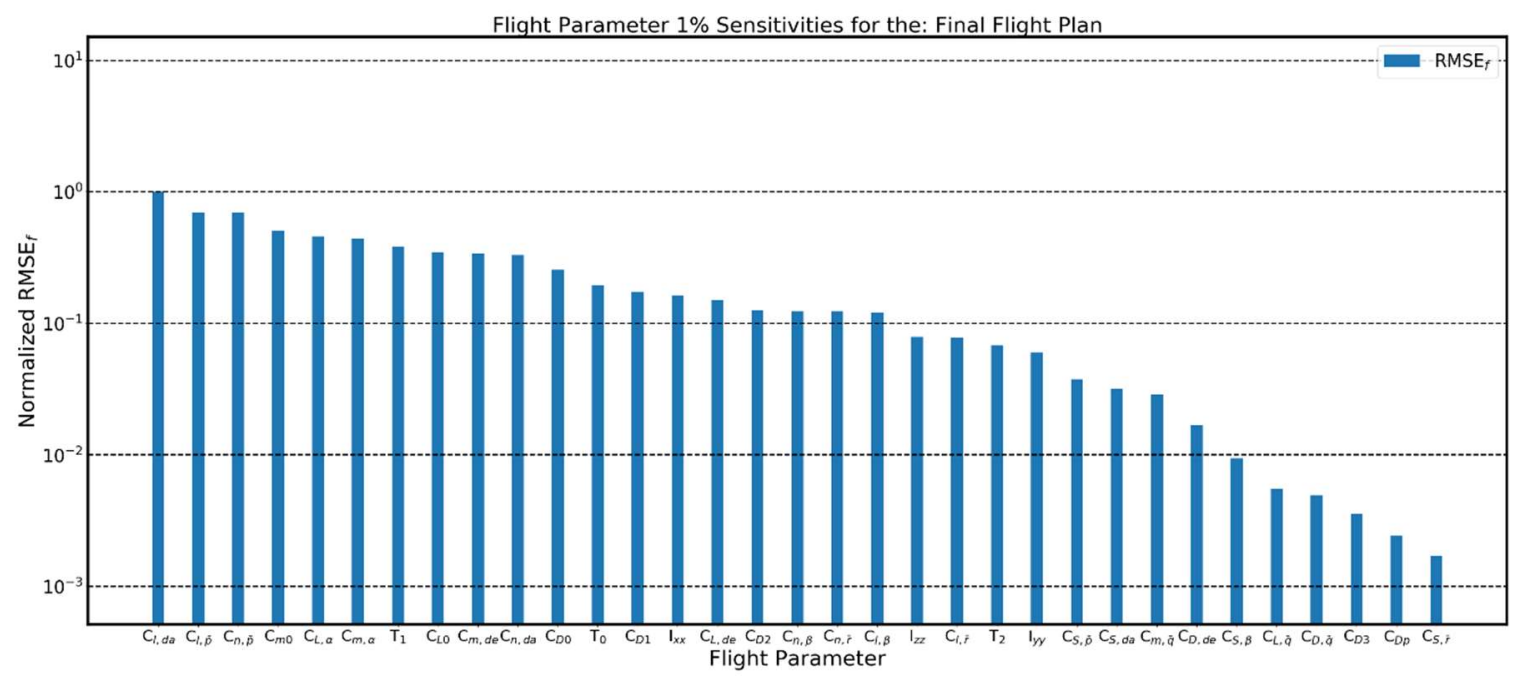

Fig. $11 R M S E_{f}$ sensitivities of the aircraft parameters with a $1 \%$ change from their original value during the final flight plan. Each parameter is normalized by the parameter with the largest $R M S E_{f}$. The values can be found in Table A.3.

Figure 11 illustrates that it may be difficult to correctly identify each parameter in the trial aircraft using this specific flight plan. For example, this flight plan should be able to clearly identify parameters such as $C_{\ell, \delta_{a}}$, and $C_{m_{0}}$. However, this flight plan may not be able to accurately identify parameters like $C_{D_{p}}$ and $C_{S, \bar{r}}$. As a reminder, Jategaonkar [5] explains that if a parameter's sensitivity is less than $10 \%$ of the largest parameter's sensitivity, it might not be identifiable.

As mentioned earlier, the literature on system identification for rudderless aircraft is limited. This is partly due to the difficulty in exciting some of the lateral modes to the point that they become dominant in the data. This is usually accomplished with rudder doublets and impulses. Since the aircraft of interest does not have a rudder to excite the less sensitive lateral parameters, we incorporated a diverse set of lateral maneuvers into the final flight plan to have more of the flight be dependent on the lateral parameters.

\section{Parameter Estimation}

There are multiple estimation methods available for predicting the parameters within a mathematical model. The three main types commonly used are equation error, output error, and filter error methods [24]. The equation error method minimizes a cost function equation defined by the inputs and outputs of the model. It is often regarded as the simplest approach to parameter estimation. This is because estimates are obtained through a set of matrix algebraic operations in one computational iteration [5]. Output error methods estimate the parameters by iteratively adjusting the parameters in order to minimize the error between the measured responses and the estimated responses. This generally leads to a nonlinear optimization problem where the computational cost is higher than equation error methods. Additionally, due to the nonlinear non-convex nature of the output error methods, they often display multiple local minima within the solution space [25]. The output error method is the most commonly used in the time-domain for parameter estimation. Filter error methods are the most complex of the three. Filter error methods are a combination of the output error method with some applied filtering techniques to account for error and noise in the data sets. They are generally combined with Kalman filters which results in higher computational cost than standard output error methods [25]. The work presented in this paper employs an output error method for estimating the model parameters.

\section{A. $R M S E_{f}$ Behavior for Individual Parameters}

Visualizing model behavior can be helpful in determining what type of optimization technique may be needed to accurately estimate the parameters. Since there are 33 parameters being estimated, there are 33 independent variables being considered. Since the human mind is incapable of visualizing information in 33 dimensions, it would be impossible to visualize the complete model behavior. However, we can break up the visualization by the individual 
parameters to get a sense of how well conditioned the model is for a given parameter. This is done by perturbing the parameter of interest over a range of perturbations from $-10 \%$ to $10 \%$ from the baseline value of that parameter and plotting the $R M S E_{f}^{2}$. The $R M S E_{f}$ is squared to make linear trends appear parabolic for future gradient estimation schemes. The results of this study are displayed in Fig. 12.

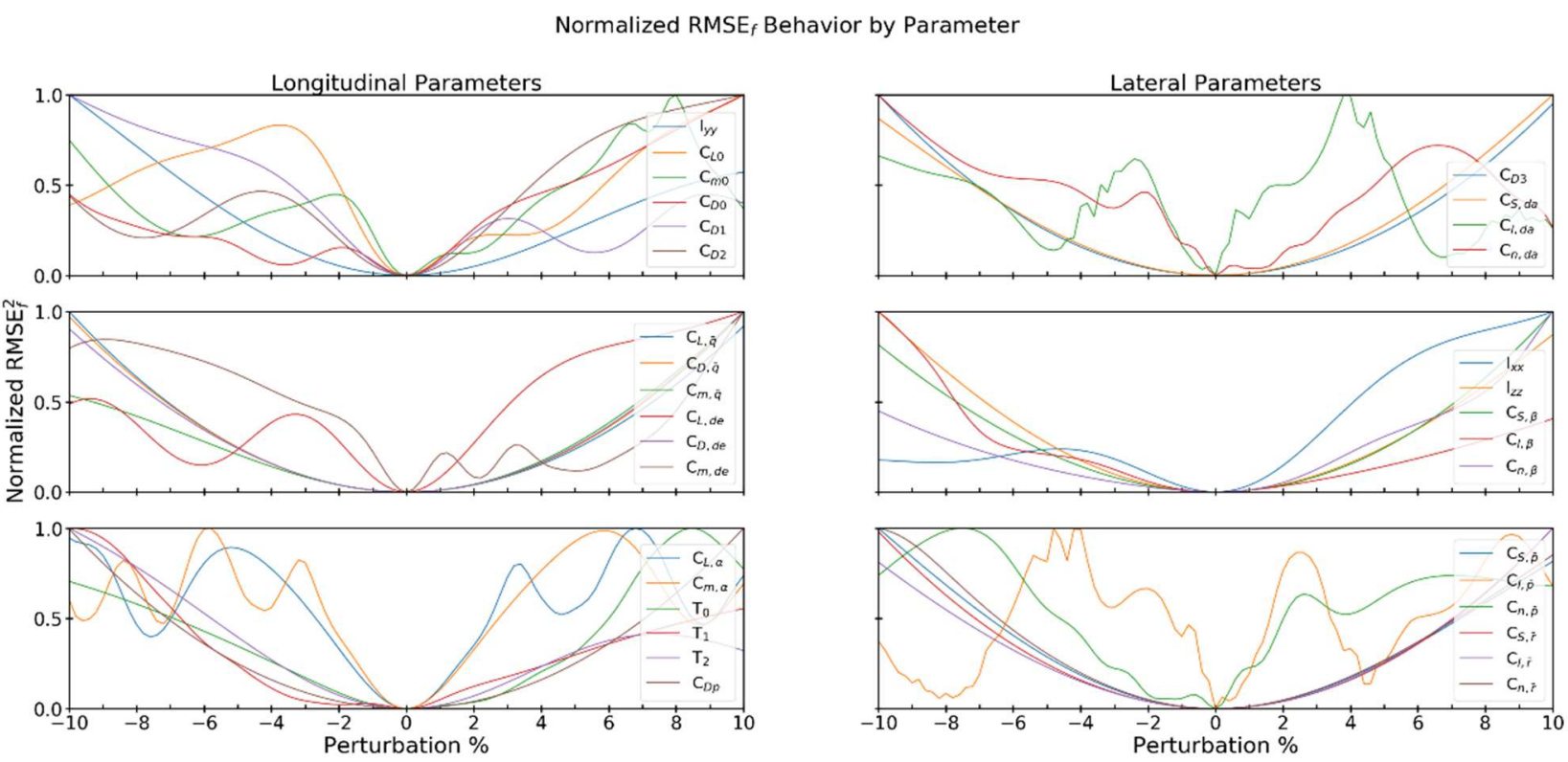

Fig. $12 R M S E_{f}^{2}$ behavior for each parameter. Each curve is normalized by the highest $R M S E_{f}^{2}$ achieved by that parameter over the range of perturbations. The parameters are perturbed from $-10 \%$ to $10 \%$ of the baseline aircraft parameter value.

From the results in Fig. 12, it is clear the model is ill-conditioned to estimate certain parameters due to the erratic behavior and abundance of local minima. Interestingly, a few of these parameters are the ones previously thought to be readily estimated from the final sensitivity plot in Fig. 11. Parameters like $C_{\ell, \delta_{a}}, C_{\ell, \bar{p}}$, and $C_{n, \bar{p}}$ returned much higher $R M S E_{f}^{2}$ values at $1 \%$ perturbations than their counterparts, which accounts for their high sensitivity shown in Fig. 11. However, the non-parabolic nature of these parameters indicates that it will be difficult to accurately estimate the correct values using a gradient-based optimization scheme for the final flight plan.

The desired behavior of the $R M S E_{f}^{2}$ value for each parameter would be varying scaled parabolas without the local minima interferences similar to $C_{L, \bar{q}}, C_{D, \bar{q}}$, and $C_{m, \bar{q}}$. This would allow a gradient-based estimation technique to accurately estimate the parameters for this model and flight plan. Unfortunately, the abundance of local minima in certain parameters means that a gradient-based minimization technique may not return to the baseline parameter value, particularly for realistic initial guesses around $10-20 \%$ of the actual value. This may be overcome if the gradientbased minimization technique employs some random search or genetic programming techniques.

\section{B. Coupled Parameter Behavior}

As an additional step to visualizing the complex model behavior, each combination of two parameters was run through a gradient-based estimation algorithm. This was done to understand how estimating combinations of parameters may affect the ability to estimate all 33 parameters at the same time.

\section{Estimation Algorithm}

This section describes the gradient-based estimation algorithm used in this paper. Parameters are estimated through a system identification algorithm that uses a steepest-descent gradient method for minimizing the $R M S E_{f}$. The first step in the process is to record the $x_{f}, y_{f}$, and $z_{f}$ states and control inputs for the baseline aircraft as it performs the final flight plan from the sensitivity analysis using a specified flight simulator. The states from the baseline flight are representative of recorded flight data from a prototype aircraft. The trial aircraft model is flown using the same control inputs as the baseline aircraft in an optimization loop. The algorithm follows the pattern outlined in Fig. 13 until the $R M S E_{f}$ value falls below $1 \mathrm{E}-8$ or until other exit criteria is met (i.e. max iterations, etc.). 


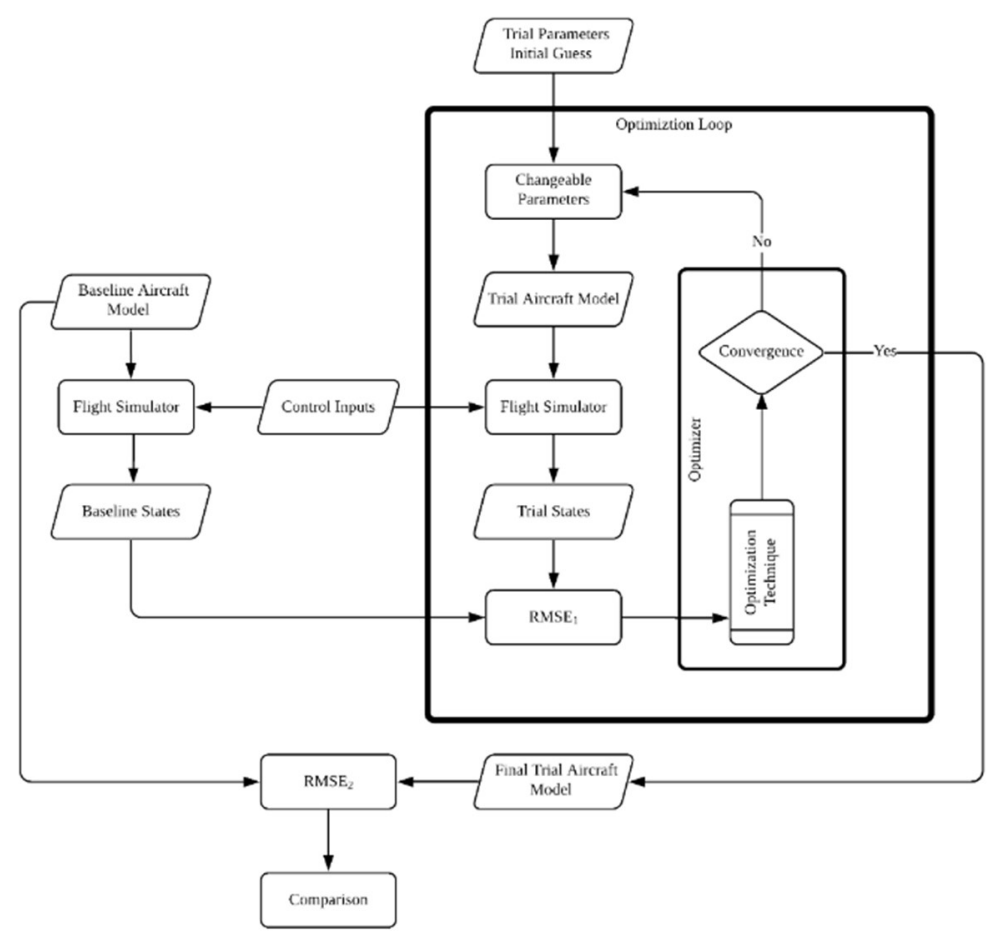

Fig. 13 Flow chart for the system identification algorithm used.

The algorithm has four main parts. The first is the baseline aircraft simulation shown at the very left of Fig. 13. It is comprised of the baseline aircraft model, a simulated flight, and the recorded baseline states. The baseline states and recorded control inputs represent the recorded data from a prototype aircraft. The second part is the main optimization loop shown in the large bold outline on the right of Fig. 13. This is the guts of the system identification algorithm. This part uses the trial aircraft model with the 33 parameters being estimated in the specified flight simulator to generate the trial states. The $R M S E_{f}$ is computed after each run of the simulator and used to help determine convergence or estimate the next guess of the estimated parameters. This $R M S E_{f}$ value given in Eq. (44) is a scalar value representative of the entire flight. Slight changes in the trial aircraft parameters can have large effects on the aircraft state. The optimization loop in the bold box seeks to reduce that value. As the loop iterates, the dotted lines in Fig. 10 will get shorter as the trial flight path converges to the baseline. The third part is the trial parameters initial guess at the top of Fig. 13. The initial guesses used for each of the 33 estimated parameters were $1 \%$ greater than the baseline parameters to see if the algorithm could return the original values from a small deviation. Lastly, the baseline aircraft parameters and the final estimated trial aircraft parameters are compared through the $R M S E_{p}$ function shown as the bottom three steps in Fig. 13. The $R M S E_{p}$ function compares the baseline aircraft parameters to the trial aircraft parameters through

$$
R M S E_{p}=\sqrt{\sum_{i=1}^{N} \frac{\left(\widetilde{\Pi}_{i}-\Pi_{i}\right)^{2}}{N}}
$$

where $N$ is the number of parameters that are being estimated, and $\widetilde{\Pi}_{i}$ and $\Pi_{i}$ are the baseline aircraft parameters and the trial aircraft parameters respectively. This scalar value is a measure of how well the algorithm was able to estimate each of the flight parameters. Mathematically, the algorithm solves

$$
\min _{\Pi_{i}} \sum_{i=1}^{\eta} \sum_{j=7}^{9} \frac{\left(\tilde{s}_{i j}-s_{i j}\right)^{2}}{\eta \times 3}
$$

where $\eta$ is the number of time-steps in the state files, $\tilde{s}_{i j}$ is the baseline Earth-fixed points, and $s_{i j}$ is the trial Earthfixed points. 


\section{Coupled Parameter Results}

Every possible pair of the 33 parameters was run through the estimation algorithm using the L-BFGS-B minimization technique in the "minimize" function of the Scipy.Optimize ${ }^{\dagger \dagger}$ class in Python. The default settings for the L-BFGS-B method were used. The results of the coupled parameter behavior are presented in Fig. 14 .

a)

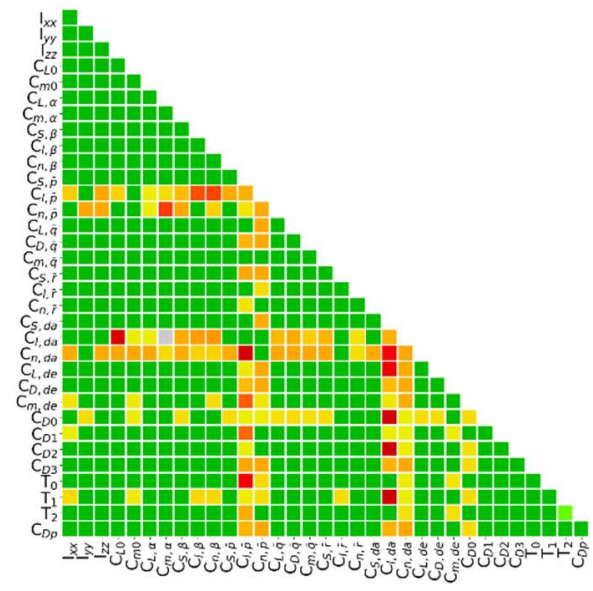

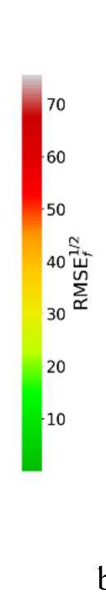

b)

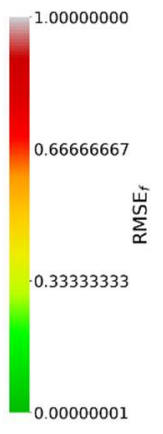

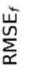

\section{1}

Fig. 14 a) Final $R M S E_{f}^{1 / 2}$ for each combination of estimated parameters given a $1 \%$ perturbation from the baseline. The results on the major diagonal represent each attempt to estimate an individual parameter. b) $\boldsymbol{R M S E _ { f }}$ for each combination with all the combinations' results greater than 1 set equal to one.

The results from Fig. 14 a) are displayed as the $R M S E_{f}^{1 / 2}$ for visual purposes only. The actual estimation technique uses $R M S E_{f}^{2}$ during the minimization technique. These results demonstrate that the same parameters that are illconditioned for estimation from the individual parameter analysis continue to negatively impact the estimation results. Figure $14 \mathrm{~b}$ ) illustrates how well the $R M S E_{f}$ of certain pairs of parameters go to zero. This is done by setting all the $R M S E_{f}$ values that are greater than one equal to one. From Fig. $14 \mathrm{~b}$ ) we see that nearly all the pairs of parameters that were successfully estimated returned $R M S E_{f}$ values near $1 \mathrm{E}-8$, with the exception of $I_{x x}$ and $C_{D 3}$. If we remember that the $R M S E_{f}$ is a measure of the difference between the Earth-fixed states of the baseline aircraft and the trial aircraft, then Fig. $14 \mathrm{~b}$ ) presents additional information. It shows that of the pairs of parameters that were able to be estimated, the overall distance between the two aircraft was less than $0.000001 \mathrm{ft}$. through the entire flight. It is important to note that this level of scrutiny and accuracy is only useful in the world of simulation. This result does not claim that we can accurately estimate the parameters to within 0.000001 of the true value. This is because we can't measure the position of a prototype aircraft to within $0.000001 \mathrm{ft}$. However, we can scrutinize the simulation to even smaller values since machine precision is roughly 12-13 decimal places. Therefore, the purpose for highlighting the $0.000001 \mathrm{ft}$. difference is to instill confidence in the numerical techniques and their ability to estimate. Additionally, Fig. 14 also shows that certain combinations of well-conditioned parameters may not be estimated together. For example, $C_{m_{0}}$ and $T_{1}$ displayed well-conditioned behavior for parameter estimation from Fig. 12. However, when combined, the estimation algorithm struggled to minimize the $R M S E_{f}^{2}$ function. This may indicate that while they are individually well-conditioned for estimation, the dynamics governed by these two parameters may be ill-conditioned for a gradient-based minimization technique using the final flight plan.

Some possible solutions to this problem might include using an alternative flight plan with different maneuvers or using a different optimization scheme to minimize the $R M S E_{f}^{2}$, such as a genetic algorithm. Furthermore, some parameters may be removed from the analysis to reduce the number of competing variables. Finally, it is important to remember that the $R M S E_{f}^{2}$ only compared the Earth-fixed positions of the baseline aircraft and the trial aircraft. It may be necessary to compare all 13 states from Eqs. (39) - (42) given by the $R M S E_{a}$ from Eq. (43). The issues revealed by this analysis may indicate why system identification is conducted the way it is today. Which entails breaking the process into multiple, smaller, flights that are each dedicated to identifying a specific set of parameters.

One of the limitations to this analysis is that it only portrays how pairs of parameters behave together. It is entirely possible that a given set of well-conditioned parameters may become ill-conditioned when combined with another set of parameters. However, the estimation algorithm only had to be run 561 different times in order to account for each

\footnotetext{
${ }^{\dagger}$ https://docs.scipy.org/doc/scipy/reference/generated/scipy.optimize.minimize.html
} 
paired combination of the 33 parameters and the individual parameter cases. In contrast, an analysis of all possible combinations of the 33 parameters would require the estimation algorithm to be run approximately 4.7E36 different times which is not feasible with today's technology. Additionally, it is impossible for the human brain to visualize this $33 \times 33 \times 33$ array of possibilities.

\section{Gradient-Based Optimization for Parameter Estimation}

With some visual insight to the challenge of estimating all 33 parameters with a single flight, we expected the gradient-based optimization scheme to struggle with correctly estimating all 33 flight parameters using the final flight plan from the sensitivity analysis. Originally, the algorithm used the Python based Pylot simulator as the flight simulator responsible for computing the estimated behavior of the trial aircraft in each iteration. Since the real-time duration of the flight was 13 minutes long, Pylot could run the final flight in computer-time in approximately 50 seconds. This becomes costly in time because there are 33 parameters. At each iteration, the gradient must be estimated using a finite difference scheme for each parameter. This means, in order to determine the direction of the next step in the loop, the flight had to run 67 times, twice for each parameter's gradient, and once to determine where the function currently is. As a result, one iteration of the L-BFGS-B method took nearly an hour. Initial trials using the Pylot simulator ran for over a week without finishing. This led to efforts to speed up the simulator and the gradient calculation. Python is a scripted language and runs inherently slower than alternative compiled languages. As a result, a custom Fortran simulator code was adapted to match the mathematical model presented in section III. For a given set of control inputs, both Pylot and the Fortran simulator produced the same sequence of aircraft states to machine precision. The faster compiled Fortran code was able to reduce the computer-time required to run a single flight from 50 seconds to less than half a second. Additionally, the function responsible for computing the gradient at each iteration was adapted to handle parallel processing. This allowed the code to take full advantage of the number of processers available on an arbitrary machine. Once these enhancements had been made, the final code used the Fortran simulator in conjunction with the parallel processing gradient. Running on a machine with 64 cores, this allowed the gradient to be computed in less than a full second. As a result of these improvements, the entire algorithm finished in approximately 3,500 seconds, or less than an hour.

All 33 parameters were estimated using the algorithm depicted in Fig. 13. With the only difference being that $N=$ 33 in Eq. (54). Each parameter's initial guess was started at $1 \%$ greater than the corresponding parameter from the baseline aircraft. Additionally, in order to prevent Scipy's minimize function from guessing unrealistic parameter values, the parameter guesses were bounded by $\pm 10 \%$ of the initial guess. This was necessary because the integration schemes in the Pylot and Fortran simulators struggled with unrealistically large or small parameter guesses from the optimizer. As expected, the algorithm struggled to minimize Eq. (55) and therefore, struggled to correctly estimate the parameters. Even at an initial guess of $1 \%$ from the baseline values, and guesses bounded at $\pm 10 \%$ of the initial value, the returned $R M S E_{p}=0.7 \%$.

\section{Parameter Estimation Results Discussion}

The results from the parameter estimation section yielded some insight to the behavior of the mathematical model for the final flight plan determined in the sensitivity analysis. First, we were able to determine that the parameters $C_{\ell, \delta_{a}}, C_{n, \delta_{a}}, C_{\ell, \bar{p}}$, and $C_{n, \bar{p}}$ displayed erratic behavior that made the model ill-conditioned for accurately estimating these parameters. From Fig. 12, we also found the presence of multiple local minima in the $R M S E_{f}$ behavior. This could be a function of the model structure or of the flight plan used in the study. Second, we were able to show that when the ill-conditioned parameters were paired with other parameters it became difficult to accurately estimate that pair of parameters as expected. Additionally, Fig. 14 shows that while some of the parameters were individually wellconditioned for parameter estimation, the combination of well-conditioned parameter pairs did not ensure accurate estimation of the pair. This can be seen with the parameters $C_{m_{0}}$ and $T_{1}$. Both parameters displayed well-conditioned behavior on their own, but when they were paired together, they became ill-conditioned for estimation. This could indicate a competing, coupled behavior in the dynamic model for these parameters. Third, as expected, the gradientbased approach for parameter estimation struggled to accurately estimate all 33 parameters with a single flight. The gradient-based estimation algorithm was rarely able to improve the initial percent difference of the initial guess at the start of the algorithm. For example, if the initial guesses were $1 \%$ deviations from the baseline aircraft parameters, the $R M S E_{p}$, which started at 0.01 , was only reduced to 0.007 after the optimization had finished. The minimization was also conducted using a direct-search, simplex minimization scheme. The Nelder-Mead method is a modified simplex method available in Scipy's minimize function. The estimation algorithm took 2 hours and 45 minutes to terminate as opposed to the 58 minutes from the L-BFGS-B method. The Nelder-Mead method was also started with initial guesses 
that were $1 \%$ deviations from the baseline parameters. The final $R M S E_{p}$ from the Nelder-Mead was increased from 0.01 to 0.0103 with some parameters getting closer to the baseline value and others being estimated at values more than the initial $1 \%$ deviation. Lastly, it is apparent that a gradient-based optimization scheme may not be suited to estimate all 33 parameters using a single flight due to the pervasiveness of the local minima in certain parameters.

\section{Conclusion}

A white box model was implemented in the time-domain to try and estimate 33 aircraft parameters for a rudderlessaircraft. The parameters were estimated using a single flight that is designed to excite as many dynamic modes as possible, with an emphasis on lateral modes. The final flight plan was designed from a sensitivity analysis where 16 different flight maneuvers were analyzed to determine which parameters were sensitive to a given flight maneuver. Sensitivity was defined by Eq. (44) which is a measure of the overall distance between the trial aircraft and the baseline aircraft in the Earth-fixed coordinate system. Without a rudder to enter, and remain, in an uncoordinated flight configuration, certain lateral parameters like $C_{S, \delta_{\alpha}}, C_{S, \beta}$, and $C_{S, \bar{r}}$ were difficult to excite. Ten maneuvers were selected for the final flight plan. The maneuvers were selected and ordered to try and extract all 33 parameters. Lateral maneuvers were interspersed throughout the flight to increase the significance of the lateral parameters throughout the flight. The combination of maneuvers proved to increase the overall sensitivity of each parameter relative to the parameter with the highest sensitivity. Additionally, a closed form approximation for aircraft natural frequencies is presented following Phillips [7] derivation.

The $R M S E_{f}$ behavior for each parameter was visually represented. From Fig. 12 it was observed that the lateral parameters, $C_{\ell, \delta_{a}}, C_{\ell, \bar{p}}$, and $C_{n, \bar{p}}$, were ill-conditioned for a gradient-based parameter estimation due to the abundance of local minima. Interestingly, these were some of the parameters that demonstrated the highest levels of parameter sensitivity to the final flight plan in Fig. 11.

An iterative output error method was implemented within a gradient-based minimization algorithm to estimate the flight parameters. A coupled parameter study was conducted where every possible pair of the 33 parameters was run through the estimation algorithm to gain additional insight into the behavior of the estimation problem. The results from Fig. 14 demonstrated that the ill-conditioned parameters negatively affected the estimation of most parameter pairs they were included in. Additionally, it was shown that when some of the individual well-conditioned parameters were paired together, they became ill-conditioned for estimation. This behavior may extend to other larger groups of parameters. The pairs of parameters that were accurately estimated in Fig. 14 were able to be estimated accurately enough to reduce the overall error between the baseline and trial aircraft Earth-fixed position to less than 1E-6 feet. Again, it should be noted that this level of scrutiny and accuracy is only useful in the world of simulation. This result does not claim that we can accurately estimate the parameters to within 0.000001 of the true value. However, we can scrutinize the simulation to this level since machine precision is roughly 12-13 decimal places. Therefore, the 0.000001 $\mathrm{ft}$. difference is a measure of the confidence of the numerical method presented in this text.

Finally, the L-BFGS-B method in Scipy's minimize function was used to estimate all 33 parameters using the final flight plan from the sensitivity analysis. The gradient-based method struggled to accurately estimate the parameters with initial guesses that were only $1 \%$ different from the baseline parameters. This could be due to the abundance of local minima within the solution space. Some possible solutions for future work may be to incorporate a genetic algorithm that can move around different local minima in the solution space and locate the optimal value. Additionally, certain parameters may be competitively coupled, prohibiting effective joint estimation. It is also important to note that the results from the estimation study are specific to the aerodynamic model, derived at the beginning of the paper, and to the final flight plan developed in the sensitivity analysis. Further work and testing could be done with maneuver development and model improvements. This insight may reveal why aircraft system identification is conducted in a series of short flight maneuvers, each dedicated to estimating a small set of parameters at a time.

\section{Acknowledgements}

This work was funded by the U.S. Office of Naval Research Sea-Based Aviation program (Grant No. N00014-181-2502) with Brian Holm-Hansen as the program officer. Additional funding and resources were provided by the Space Dynamics Laboratory TERRA IR\&D with Jonathan Haws as the project manager. 


\section{References}

[1] Venkataraman, R., and Seiler, P. "System Identification for Small, Rudderless, Fixed-Wing Unmanned Aircraft.” Journal of Aircraft, Vol. 56 No. 3, 2019, pp. 1126-1134. Doi: 10.2514/1.C035141

[2] Tischler, M. B., and Remple, R. K. Aircraft and Rotorcraft System Identification, 2nd ed., AIAA, Reston, VA 2012.

[3] Pappalardo, C.M., and Guida, D. "A Time-Domain System Identification Numerical Procedure for Obtaining Linear Dynamical Models of Multibody Mechanical Systems." Archive of Applied Mechanics, 88, 2018, pp 1325-1347. Doi: 10.1007/s00419-018-1374.

[4] Nielsen, H. A., and Madsen, H. "Modeling the Heat Consumption in District Heating Systems Using a GreyBox Approach.” Energy Buildings. 38, 2006, pp. 63-71, Doi: 10.1016/j.enbuild.2005.05.002.

[5] Jategaonkar, R. V. Flight Vehicle System Identification: A Time Domain Methodology, 1st ed., Vol. 216, AIAA, Reston, VA, 2006.

[6] Cook, M. V., "Flight Dynamics Principles,” 2nd ed., Elsevier, 2007, Chaps. 4, 5, 6, 7.

[7] Phillips, W. F., Mechanics of Flight. 2nd ed., Wiley, Hoboken, NJ, 2010.

[8] Smith, G. D., and Bixler, B. M., and Babcock, J. T., and Osteroos, R. K., and McLaughlin, T. E., and Tischler, M. B. "System Identification of the ICE/SACCON UAS Aircraft." AIAA SciTech 2020, Orlando, 2020. doi:10.2514/6.2020-0289.

[9] Williams, D. R., Seidel, J., Osteroos, R and McLaughlin, T. E., "NATO AVT-239 Task Group: Flight Control Derivatives using Active Flow Control Effectors on the ICE/SACCON UAS Model," 2019. . doi:10.2514/6.2019-0043.

[10] Grauer, J. A., and Boucher, M. J. "System Identification of Flexible Aircraft: Lessons Learned from the X-56A Phase 1 Flight Tests.” AIAA SciTech 2020, Orlando 2020. doi: 10.2514/6.2020-1017

[11] Regan, C. D., and Kotikalpudi, A., and Schmidt, D. K., and Seiler, P., "mAEWing2: Initial Flight Test and System Identification of a Flexible UAV.” AIAA SciTech 2020, Orlando, FL, 2020.

[12] Ljung, Lennart., and Glover, Keith., "Frequency Domain Versus Time Domain Methods in System Identification," Automatica the Journal of IFAC, Vol. 17 No. 1, 1981, pp. 71-86.

[13] Tischler, M. B., et al., "Time and Frequency-Domain Identification and verification of BO 105 Dynamic Models,” Fifteenth European Rotorcraft Forum, Amsterdam, 1989.

[14] Pintelon, Rik., and Schokens, Johan., System Identification: A Frequency Domain Approach, $2^{\text {nd }}$ ed., WileyIEEE Press, Hoboken, NJ, 2012.

[15] Schoukens, J., and Pintelon, R., and Rolain, Y., "Time domain identification, frequency domain identification. Equivalencies! Differences?,” American Control Conference, Boston, MA, 2004.

[16] Phillips, W. F., and Snyder, D. O. "Modern Adaptation of Prandtl's Classic Lifting-Line Theory." Journal of Aircraft, Vol. 37 No. 4, 2000, pp. 662-670. doi:10.2514/2.2649.

[17] Reid, J. T., and Hunsaker, D. F., "A General Approach to Lifting-Line Theory, Applied to Wings with Sweep." AIAA SciTech 2020, Orlando, 2020. 
[18] Klein, V., and Morelli, E. A., "Mathematical Model of an Aircraft," Aircraft System Identification Theory and Practice, AIAA Education Series, 2006.

[19] Kimberlin, R.D., Flight Testing of Fixed-Wing Aircraft, AIAA, Reston, VA, Sept. 2003.

[20] Lee, R. E., Jr., “Handling Qualities,” AGARD-AR-300 Vol. 14, Paper No. 15, Sept. 1995.

[21] Gallagher, G.L., Higgins, L. B., Khinoo, L. A., and Pierce, P. W., "Fixed Wing Performance," Naval Test Pilot School Flight Test Manual, Report USNTPS-FTM-No. 108, Sept. 1992.

[22] Morelli, Eugene, A., "In-Flight System Identification," AIAA paper 98-4261, NASA Technical Reports Server, Jan. 1998.

[23] Koehler, R. and Wilhelm, K.. “Auslegung von Eingangssignalen für die Kennwertermittlung,” DFVLR-IB 15477/40, Dec. 1977 (German language)

[24] Raol, J. R., Girija, G., and Singh, J., Modelling and Parameter Estimation of Dynamic Systems, The Institution of Engineering and Technology, London, UK, 2004.

[25] Tomita, Y., Damen, AD A. H., and Van Den Hof, P. M. J., "Equation error versus output error methods," Ergonomics, Vol. 35, Issue 5-6, pp. 551-564, doi: 10.1080/00140139208967836. 


\section{Appendix}

Table A.1 $R M S E_{f}$ values for each of the 33 parameters for a short period 3-2-1-1 flight maneuver. Each value is normalized by the largest $R M S E_{f}$ for the flight. In this case, $C_{m, \alpha}$ with a value of 4.47 .

\begin{tabular}{cccc|cccc}
\hline \hline & \multicolumn{3}{c|}{ Longitudinal } & \multicolumn{4}{|c}{ Lateral } \\
\hline$C_{m, \alpha}$ & 1.0000 & $C_{D 1}$ & 0.0882 & $C_{\ell, \beta}$ & 0.0027 & $I_{Z z}$ & 0.0001 \\
$C_{L, \alpha}$ & 0.9919 & $C_{D 2}$ & 0.0771 & $C_{n, \beta}$ & 0.0027 & $C_{S, \beta}$ & $>1 \mathrm{e}-04$ \\
$C_{m, \delta_{e}}$ & 0.5035 & $T_{2}$ & 0.0282 & $C_{\ell, \delta_{a}}$ & 0.0018 & $C_{S, \bar{p}}$ & $>1 \mathrm{e}-04$ \\
$C_{m_{0}}$ & 0.4952 & $I_{y y}$ & 0.0112 & $I_{x x}$ & 0.0014 & $C_{S, \bar{r}}$ & $>1 \mathrm{e}-04$ \\
$T_{0}$ & 0.4142 & $C_{m, \bar{q}}$ & 0.0059 & $C_{n, \delta_{a}}$ & 0.0011 & $C_{S, \delta_{a}}$ & $>1 \mathrm{e}-04$ \\
$T_{1}$ & 0.3052 & $C_{D, \delta_{e}}$ & 0.0058 & $C_{n, \bar{r}}$ & 0.0010 & $C_{D 3}$ & $>1 \mathrm{e}-04$ \\
$C_{L_{0}}$ & 0.2801 & $C_{D P}$ & 0.0013 & $C_{\ell, \bar{r}}$ & 0.0006 & & \\
$C_{L, \delta_{e}}$ & 0.1785 & $C_{L, \bar{q}}$ & 0.0006 & $C_{\ell, \bar{p}}$ & 0.0006 & & \\
$C_{D 0}$ & 0.1433 & $C_{D, \bar{q}}$ & 0.0001 & $C_{n, \bar{p}}$ & 0.0002 & & \\
\hline \hline
\end{tabular}

Table A.2 $R M S E_{f}$ values for each of the 33 parameters for a barrel roll flight maneuver. Each value is normalized by the largest $R M S E_{f}$ for the flight. In this case, $C_{\ell, \delta_{a}}$, with a value of 264.0 .

\begin{tabular}{clll|lcll}
\hline \hline \multicolumn{3}{c}{ Longitudinal } & \multicolumn{5}{|c}{ Lateral } \\
\hline$C_{m_{0}}$ & 0.2426 & $C_{D 1}$ & 0.0223 & $C_{\ell, \delta_{a}}$ & 1 & $C_{S, \delta_{a}}$ & 0.0034 \\
$C_{L, \alpha}$ & 0.1859 & $C_{D 2}$ & 0.0216 & $C_{\ell, \bar{p}}$ & 0.9459 & $C_{\ell, \bar{r}}$ & 0.0016 \\
$C_{m, \alpha}$ & 0.1732 & $I_{y y}$ & 0.0208 & $C_{n, \delta_{a}}$ & 0.3146 & $C_{S, \beta}$ & 0.0009 \\
$C_{L_{0}}$ & 0.1370 & $T_{2}$ & 0.0049 & $C_{n, \bar{p}}$ & 0.2549 & $C_{n, \bar{r}}$ & 0.0008 \\
$C_{m, \delta_{e}}$ & 0.0683 & $C_{m, \bar{q}}$ & 0.0031 & $C_{n, \beta}$ & 0.0544 & $C_{D 3}$ & 0.0003 \\
$T_{0}$ & 0.0477 & $C_{D, \delta_{e}}$ & 0.0027 & $I_{x x}$ & 0.0466 & $C_{S, \bar{r}}$ & 0.0001 \\
$T_{1}$ & 0.0416 & $C_{L, \bar{q}}$ & 0.0006 & $C_{\ell, \beta}$ & 0.0445 & & \\
$C_{D 0}$ & 0.0254 & $C_{D, \bar{q}}$ & 0.0004 & $I_{z z}$ & 0.0193 & & \\
$C_{L, \delta_{e}}$ & 0.0236 & $C_{D P}$ & 0.0002 & $C_{S, \bar{p}}$ & 0.0041 & & \\
\hline \hline
\end{tabular}

Table A.3 $R M S E_{f}$ values for each of the 33 parameters for the final flight plan. Each value is normalized by the largest $R M S E_{f}$ for the flight. In this case, $C_{\ell, \delta_{a}}$, with a value of 3863.64.

\begin{tabular}{cccc|cccr}
\hline \hline \multicolumn{3}{c|}{ Longitudinal } & \multicolumn{5}{|c}{ Lateral } \\
\hline$C_{m_{0}}$ & 0.5030 & $C_{L, \delta_{e}}$ & 0.1498 & $C_{\ell, \delta_{a}}$ & 1 & $C_{\ell, \bar{r}}$ & 0.0777 \\
$C_{L, \alpha}$ & 0.4548 & $C_{D 2}$ & 0.1252 & $C_{\ell, \bar{p}}$ & 0.6962 & $C_{S, \bar{p}}$ & 0.0375 \\
$C_{m, \alpha}$ & 0.4434 & $T_{2}$ & 0.0678 & $C_{n, \bar{p}}$ & 0.6958 & $C_{S, \delta_{a}}$ & 0.0316 \\
$T_{1}$ & 0.3818 & $I_{y y}$ & 0.0596 & $C_{n, \delta_{a}}$ & 0.3296 & $C_{S, \beta}$ & 0.0093 \\
$C_{L_{0}}$ & 0.3487 & $C_{m, \bar{q}}$ & 0.0288 & $I_{x x}$ & 0.1629 & $C_{D 3}$ & 0.0035 \\
$C_{m, \delta_{e}}$ & 0.3376 & $C_{D, \delta_{e}}$ & 0.0168 & $C_{n, \beta}$ & 0.1234 & $C_{S, \bar{r}}$ & 0.0017 \\
$C_{D 0}$ & 0.2552 & $C_{L, \bar{q}}$ & 0.0055 & $C_{n, \bar{r}}$ & 0.1231 & & \\
$T_{0}$ & 0.1939 & $C_{D, \bar{q}}$ & 0.0049 & $C_{\ell, \beta}$ & 0.1199 & & \\
$C_{D 1}$ & 0.1738 & $C_{D_{P}}$ & 0.0024 & $I_{z Z}$ & 0.0787 & & \\
\hline \hline
\end{tabular}

